\title{
Contextual influences on interactive processing: Effects of discriminability, quantity, and uncertainty
}

\author{
ROBERT D. MELARA and JEFFREY R. W. MOUNTS \\ Purdue University, West Lafayette, Indiana
}

\begin{abstract}
Three contextual factors-(1) the discriminability of stimuli in pitch, (2) the number of stimuli differing in pitch, and (3) the uncertainty regarding which stimuli or tasks would appear-were manipulated as subjects performed speeded loudness classifications in each of six experiments. The magnitude of Garner interference and effects of congruity were used to gauge the degree of interactive processing. Enhancing pitch discriminability caused monotonic increases in interference and congruity. Stimulus-task uncertainty mediated the changes in Garner interference wrought by increased discriminability. Uncertainty also caused a surprising shift in congruity from strongly positive to strongly negative as uncertainty grew. Increasing stimulus quantity lowered interference, but had inconsistent effects on congruity. Regression analyses suggested that, collectively, these three contextual variables underlie most failures of selective attention in speeded classification.
\end{abstract}

The speeded classification paradigm, described and popularized by Garner (1974), has been used extensively to study how irrelevant variation along one perceptual dimension (e.g., auditory pitch) affects observers' speed and accuracy in classifying values along a second, relevant dimension (e.g., auditory loudness). In its basic form, the paradigm comprises two conditions: (1) baseline, in which the irrelevant dimension is held constant; and (2) filtering, in which the relevant and irrelevant dimensions are varied orthogonally. If observers' performance at classifying values on the relevant dimension is worse in filtering than at baseline-a result called Garner interference (Pomerantz, 1986)-then the two dimensions are said to interact. Garner interference reflects an inability to focus exclusively on the relevant dimension; thus, dimensional interaction is defined through a failure of selective attention. If speeded classification yields no Garner interference, the dimensions are said to be separable.

In the vast majority of studies carried out with speeded classification, only two levels of the irrelevant dimension have been tested. Rarely have more than two levels been included in a filtering task, and rarely has the physical difference between the two levels been manipulated systematically (see Lockhead, 1992). Typically, the experimenter's sole interest is in whether any Garner interference appears in classification, with the goal of deciding whether a given pair of dimensions interacts or not.

This research was supported by National Institutes of Health Grant NS28617 to R.D.M. We thank Neil A. Macmillan, Wendell R. Garner, W. Todd Maddox, and Gregory R. Lockhead for their superb comments and suggestions on an earlier version of this manuscript. We thank Anthony Gerdeman for his assistance with several preliminary experiments. Address all correspondence to R. D. Melara, Department of Psychological Sciences, Purdue University, West Lafayette, IN 47907.
A substantial amount of evidence suggests; however, that dimensional separability is not an all-or-none matter (Carvellas \& Schneider, 1972; Chipman \& Carey, 1975; Demany, 1983; Foard \& Kemler Nelson, 1984; Smith \& Kemler Nelson, 1984; Zagorski, 1975). Rather, it is becoming clear that certain conditions make the influence of one source of information on another more or less likely (Melara, Marks, \& Lesko, 1992). Accordingly, a shift in research emphasis has begun, away from simple yes-no decisions, and toward identification of what conditions enhance or reduce the possibility and/or degree of interactive processing between dimensions.

Accompanying this shift is a growing interest in the effects of stimulus context on psychophysical and perceptual judgments (e.g., Lockhead, 1992; Marks, 1988, 1992; Melara \& Marks, 1990a). The conjecture is that values from one stimulus dimension may actually alter how values from another stimulus dimension are perceived. Marks (1988, 1992; Algom \& Marks, 1990; Marks \& Warner, 1991) has provided several important demonstrations. Marks's (1988) subjects gave magnitude estimates of loudness to sounds of varying intensity. A stimulus context was created by presenting the softer sounds at a low pitch $(500 \mathrm{~Hz})$, the louder sounds at a high pitch $(2500 \mathrm{~Hz})$, and sounds of medium loudness at both pitches. A second stimulus context was then created by reversing this situation. Subjects' estimates of loudness were powerfully determined by these contexts: A $500-\mathrm{Hz}$ tone at $60 \mathrm{~dB}$ was judged as loud as a $2500-\mathrm{Hz}$ tone of $57 \mathrm{~dB}$ in the first context, but as loud as a $2500-\mathrm{Hz}$ tone at $40 \mathrm{~dB}$ in the second context.

Further investigations have revealed that these so-called slippery context effects are automatic (Marks, 1992), acting on loudness perceptions themselves, and not merely 
on the numerical judgments of loudness (Schneider \& Parker, 1990). Demonstrations like these underscore the power of context in modifying psychophysical judgments. Accordingly, Lockhead (1992) has decried traditional approaches to psychophysics, criticizing them for failing to incorporate such contextual influences into explanatory models.

\section{Lower Order and Higher Order Contextual Relations}

In the present study, we investigate context effects in speeded classification. In our view, interactive processing in multidimensional stimulus tasks is itself a context effect, originating in the contextual relations established by dimensional variation. Specifically, we claim that the nature of trial-to-trial changes along the irrelevant dimension constrains humans' ability to resolve values along the relevant dimension. Different contextual relations are created through the different patterns of irrelevant variation possible in a filtering task. These relations alter in specific ways the responses to relevant attributes, making them easier or more difficult to identify, relative to the same attributes in a baseline task in which the irrelevant dimension is held constant. Hence, the context established in filtering controls the degree of selective attention failure (i.e., the difference in performance from baseline) and, by inference, the degree of interactive processing.

At present, we are unable to identify with certainty the processing loci of the hypothesized context effects: Context may operate on perceptions (Marks, 1992), decisions (Ashby \& Townsend, 1986; Gravetter \& Lockhead, 1973; Maddox, 1992), memories of previous stimuli or responses (Durlach \& Braida, 1969; Lockhead, 1992), the competition between responses (Eriksen, O'Hara, \& Eriksen, 1982; Redding \& Tharp, 1981), or on still other aspects of classificatory behavior. The aim of the present research is instead to document, more extensively than hitherto, the empirically observed relations that exist between irrelevant context and selective attention, as a first step toward a better theoretical understanding of human failures in selective attention.

We tested the dimensions of auditory loudness and pitch. The subjects' task was always the same: Make one response to any "soft" (i.e., 60-dB) sound, and another response to any "loud" (i.e., 70-dB) sound. The subjects were instructed to ignore any changes in pitch, which were always irrelevant to the task. The stimulus context was created through pitch variation in the filtering condition. Pitch relations were established both among stimuli in a filtering task and among filtering tasks in an experiment. We asked how these contextual relations affected dimensional interaction, as gauged by changes in Garner interference.

We can bifurcate the contextual manipulations of this study into two lower order variables and one higher order variable. The lower order variables are (1) discriminability and (2) stimulus quantity. We define discriminability as the perceptual difference of values along a dimension, as determined by speed of discrimination at baseline. In the present study, stimuli tested in some filtering tasks differed greatly among one another in pitch (i.e., high pitch discriminability or range), whereas stimuli in other filtering tasks differed little in pitch (i.e., low pitch discriminability or range). We compared the magnitudes of Garner interference in classifying stimuli under these different contexts of discriminability.

We define stimulus quantity as the actual number of irrelevant values presented during the classification task. In the present study, we compared performance in the typical filtering condition, in which the stimulus set contained only two possible values of pitch, with performance in other filtering conditions, in which the set contained many values of pitch.

Previous theory and research led us to expect that both pitch discriminability and stimulus quantity would affect the classification of loudness, but in opposite directions. Increases in pitch discriminability should enhance interactive effects with loudness because, for a single value of loudness, the context established by one level of pitch becomes increasingly distinct from the context established by another level of pitch (Melara \& Marks, 1990a). Unless pitch is ignored or discounted, subjects are forced to make the same response to very different sounds (e.g., high-pitched loud sounds must receive the same response as low-pitched loud sounds). And, the greater the range of pitch, the more dissimilar are sounds assigned to a single loudness response category. Thus, increases in pitch variation should disrupt subjects' ability to classify loudness. Evidence consistent with this expectation was reported recently by Lockhead (1992, Figure 6), who found that loudness classification in filtering worsens as sounds vary more in auditory frequency.

In contrast, increases in the quantity of pitch levels should reduce Garner interference in loudness classification. This prediction is perhaps counterintuitive. It rests on the idea that, for a single value of loudness, the context established by trial-to-trial changes in pitch becomes increasingly similar. In Lockhead's (1992) terms, increasing stimulus quantity enhances the likelihood of a match between perception of the present stimulus and memory of just prior stimuli (Holland \& Lockhead, 1968; see also Felfoldy, 1974).

The higher order contextual variable is called stimulustask uncertainty: the level of ambiguity that perceivers experience about the stimuli or the tasks in a given experimental situation. In the present study, uncertainty changed from experiment to experiment according to how we coordinated the two lower order factors. In particular, when either quantity or discriminability was held constant in an experiment, uncertainty was considered to be relatively low. In Experiment 2, for example, the quantity of irrelevant values varied between tasks, but the discriminability (range) of irrelevant values was identical in every task. Uncertainty was low in this situation because subjects could focus on the end points of the range$900 \mathrm{~Hz}$ and $950 \mathrm{~Hz}$-treating them as anchors that describe the stimulus set. 
Uncertainty is considered high when both quantity and discriminability are manipulated within a single experimental session. In Experiment 4, for example, the role of particular pitch values changed considerably from task to task. A frequency of $930 \mathrm{~Hz}$ might fall toward the end of the range in some tasks, but closer to the middle of the range in other tasks. Furthermore, tasks were presented in an unpredictable order, thereby giving subjects little initial information about the range and manner of variation in the irrelevant dimension for any given task. Under such uncertain contexts, we expected interference in filtering to be severe, relative to interference in the same filtering task under conditions of low uncertainty.

In each experiment, then, we examined the effects of context by comparing a baseline condition with a set of context-laden filtering conditions. Discriminability and quantity were manipulated between tasks, while uncertainty was manipulated between experiments. To the extent that one or more of these pitch contexts fails to influence observers' responses to loudness, we would expect performance to remain constant across changes in that contextual variable. Our goal is to detail the effects of each kind of context and, in so doing, to characterize more completely the nature of interactive processes.

\section{Linear Congruity: A Measure of Stimulus Effects}

Garner interference only assesses task differences in performance. To fully understand contextual influences, we must pair this task measure with a flexible measure of stimulus relations. Simple congruity effects have often been used to assess relations among Stroop-like stimuli: Subjects classify faster and more accurately when stimulus attributes match (RED printed in red) than when they mismatch (RED printed in green; see MacLeod, 1991, for a review). Importantly, analogous results obtain with auditory stimuli: Melara and Marks (1990b) found, for example, that subjects responded faster to high-pitched/loud sounds or to low-pitched/soft sounds than to high-pitched/ soft sounds or to low-pitched/loud sounds.

Unfortunately, simple congruity effects suffer a grave limitation: Congruity is only defined dichotomously, with stimulus attributes being either congruent (matching) or incongruent (mismatching). The measure is thus inappropriate whenever one varies stimulus attributes continuously, as we did in this study, because it becomes impossible to assign particular stimuli to the categories of being congruent or incongruent. We can, however, derive a more general measure, which we call linear congruity: how classification of the relevant dimension changes as a linear function of the irrelevant dimension. A detailed description of this technique appears later.

By deriving linear congruity within each task, we were able to evaluate the behavioral effects among stimuli. Linear congruity is, in principle, acutely sensitive to stimulus relations, because it directly reflects how the context established by one attribute (e.g., a high pitch) in- fluences a subject's ability to respond to another attribute (e.g., a loud sound). One aim of this study, therefore, was to determine whether our manipulations of context would affect congruity scores. If they did, we would then be able to ask whether changes in linear congruity covary with changes in Garner interference. As we shall see, linear congruity is altered strikingly by variations in lower order and, especially, in higher order contextual relations; but these changes are not always accompanied by similar changes in Garner interference.

\section{The Present Experiments}

The specific goal of the present study is to investigate the various contextual constraints that cause pitch to affect the classification of loudness. In each experiment, we explored some aspect of either or both of the lower order stimulus contexts discussed above: (1) quantity of irrelevant values and (2) relative baseline discriminability. We measured both Garner interference and linear congruity. We asked how these measures might change as a function of the two stimulus contexts, manipulated singly or jointly. Each set of lower order manipulations creates a particular context of uncertainty. We were interested in how our measures were affected by which tasks were included in any given experimental situation. One can ask, for example, about how performance in a specific task changes as a function of uncertainty. In this study, we found that higher order experimental context, together with the lower order contexts, powerfully determined how humans performed in selective attention situations.

In many of our experiments, we found that the effects of context were described well by a linear function. Although we attach no special theoretical significance to this function, we will often find it useful-for purposes of comparing results either within or among experiments-to report the slopes and intercepts from regression analyses of Garner interference and linear congruity on our contextual variables.

\section{EXPERIMENT 1 Matching Baseline Discriminations}

In every condition of every experiment of this study (after Experiment 1), the subjects' explicit task was the same: to discriminate between a $60-\mathrm{dB}$ ("soft") sound and a 70-dB ("loud") sound. Our interest was in how well this fixed difference of $10 \mathrm{~dB}$ along the relevant dimension was identified in the context of varying differences in pitch, the irrelevant dimension. But before addressing this issue, we must identify what stimulus change in pitch corresponds, on the average, to the $10-\mathrm{dB}$ change in loudness. To isolate this point of equal discriminability, we asked subjects in Experiment 1 to discriminate various pitch differences at a constant level of loudness. We compared the reaction times (RTs) to discriminate each pitch difference with the RTs to discriminate sounds differing by $10 \mathrm{~dB}$ in intensity at a constant frequency. 


\section{Method}

Subjects. Thirty undergraduates from Purdue University, 13 men and 17 women, participated in partial fulfillment of a course requirement.

Stimuli and Apparatus. The signals were sine waves generated by an Amiga 500 microcomputer, amplified by a Realistic SA-150 amplifier, and presented over Nova -40 headphones. Instructions and feedback were provided visually on a Commodore 1084A color monitor. The total stimulus set contained 21 sounds. All signals were either 60 or $70 \mathrm{~dB}$, calibrated on the A-scale of a General Radio 1565-B sound-level meter. The signal frequencies ranged from 900 to $1000 \mathrm{~Hz}$ in steps of $10 \mathrm{~Hz}$. The frequencies were calibrated by a Heath IM-2410 frequency counter and a Heath 4251 oscilloscope. At $900 \mathrm{~Hz}$, only the 70-dB signal (i.e., the intensity used for all pitch discriminations) was tested; at all other frequencies, both intensities were included in the set. Extensive pretesting was performed, to ensure that all $60-\mathrm{dB}$ sounds appeared equally loud, despite frequency, and the same was done for all 70-dB sounds.

From this set, 20 tasks were created, 10 baseline pitch discriminations and 10 baseline loudness discriminations. Two signals were used in each task. In all pitch discriminations, both signals were $70 \mathrm{~dB}$ and one of the signals was $900 \mathrm{~Hz}$. The frequency of the other signal specified the task, from 910 to $1000 \mathrm{~Hz}$ in steps of $10 \mathrm{~Hz}$. Thus, the 10 pitch differences ranged from 10 to $100 \mathrm{~Hz}$. In all loudness discriminations, one signal was $60 \mathrm{~dB}$, the other was $70 \mathrm{~dB}$, and frequency was held constant. The frequency of the two signals specified the loudness task, from 910 to $1000 \mathrm{~Hz}$ in steps of $10 \mathrm{~Hz}$. Each task contained 100 trials, with a single stimulus (sound) presented on each trial, lasting $550 \mathrm{msec}$. Each stimulus in each task was presented on an equal number of trials. Within each task, the stimuli appeared in random order.

Procedure. The subjects were tested individually in a quiet room. The subjects alternated between pitch discrimination and loudness discrimination; which dimension was discriminated first was counterbalanced across subjects. The pitch and loudness tasks were coupled into 10 pairs of tasks; in each pair, the frequency of the signal used in loudness discrimination was the higher of the two frequencies used in pitch discrimination. A Latin square specified the order of performance of the 10 pairs of tasks. The first pair was performed once as practice; the data were discarded, and then the pair was repeated.

At the beginning of each task, written instructions specific to the particular task appeared on the computer screen. Subjects were told that one of two sounds would be presented on each trial. They were asked to press one key of a Naksha dual-key mouse if the sound was high pitched (loud) in pitch (loudness) tasks, and to press the other key if the sound was low pitched (soft). The subjects were instructed to respond fast but accurately. Key assignment (high/low, loud/soft) was counterbalanced across subjects. Responses were recorded in milliseconds by a hardware timer (Wright, 1986). The subjects received visual error feedback after incorrect responses. Trials in which latency exceeded $800 \mathrm{msec}$ were repeated at a later point in the task. Tasks in which accuracy fell below $90 \%$ were repeated immediately. After each task, the subjects were informed of their overall speed and accuracy. The entire experiment lasted approximately $75 \mathrm{~min}$.

\section{Results}

Average RTs in each of the 20 tasks are depicted graphically in Figure 1 . The abscissa denotes pitch tasks by the higher frequency of the two signals and denotes loudness tasks by the frequency of both signals. The overall error rate in this experiment was $3.83 \%: 3.97 \%$ in pitch discrimination and $3.70 \%$ in loudness discrimination. The correlation between speed and accuracy, computed in this and in other experiments according to the average performance in each task for all subjects, was .91. This indicates that
RTs and error percentages were surrogate measures, at least to a first-order approximation. The results in this article will emphasize the RT measure, although both dependent variables were analyzed statistically.

As is clear from Figure 1, pitch discriminations decreased systematically as the difference from $900 \mathrm{~Hz}$ increased. An analysis of variance (ANOVA) confirmed that this change was reliable $\left[F(9,261)=24.63, M S_{\mathrm{e}}=850.90\right.$, $p<.001$ ]. RTs dropped from $432 \mathrm{msec}$ in the $10-\mathrm{Hz}$ discrimination to $348 \mathrm{msec}$ in the $100-\mathrm{Hz}$ discrimination. This effect also obtained in the analysis of error percentages $\left[F(9,261)=19.03, M S_{\mathrm{e}}=3.61, p<.001\right]$. Subjects committed the most errors in the $10-\mathrm{Hz}$ discrimination $(7.43 \%)$; by the $50-\mathrm{Hz}$ discrimination, errors had decreased to $2.5 \%$. These changes were expected, of course, because pitch discrimination eases with increases in the frequency difference.

Perhaps less expected were changes in loudness discrimination as a function of frequency. As shown in Figure 1, despite the fact that subjects always discriminated $60 \mathrm{~dB}$ from $70 \mathrm{~dB}$ in loudness tasks, there was a tendency for performance to slow with increasing frequency $[F(9,261)$ $\left.=4.30, M S_{\mathrm{e}}=715.81, p<.001\right]$. There was also a trend toward more errors at higher frequencies, although the change in accuracy did not reach statistical significance $\left[F(9,261)=1.84, M S_{\mathrm{e}}=3.68, p=.06\right]$.

Our goal was to find what pitch difference led to speed of discrimination equal to a $10-\mathrm{dB}$ difference in loudness. Because loudness discrimination was affected by the frequency constant, obviously several different points meet this objective. Indeed, for any loudness discrimination, the matched pitch difference is obtained by drawing a horizontal line from the average RT in that loudness task

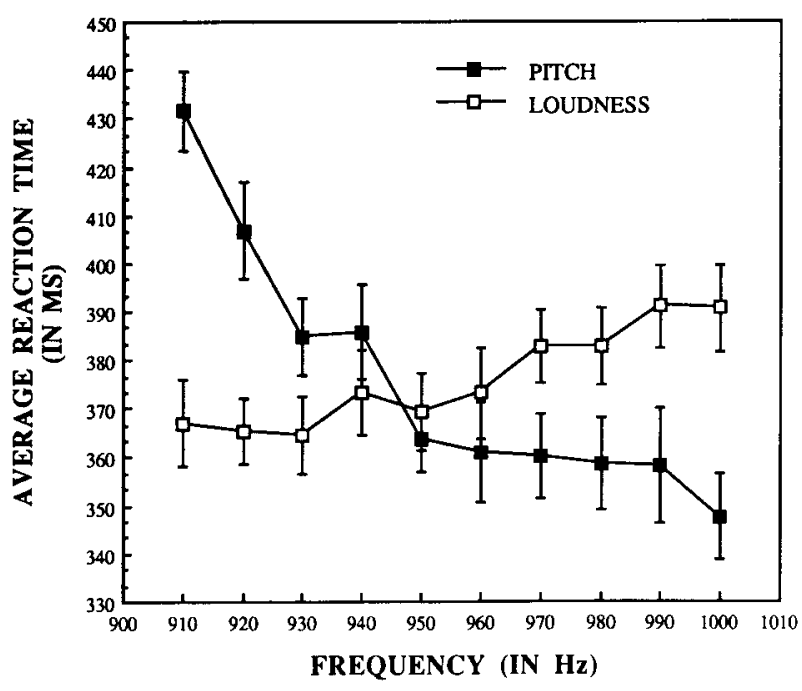

Figure 1. Average reaction time in 10 pitch discrimination tasks and 10 loudness discrimination tasks in Experiment 1. The abscissa identifies each pitch task according to the higher frequency of the two signals and identifies each loudness task according to the frequency of both signals. Note: Variability in each condition is shown by standard error bars. 
to the corresponding point on the pitch discrimination function.

To identify the average point of equal discriminability, we sought to isolate which frequency difference corresponded to the crossover point of the two discrimination functions (see Figure 1). We fit each discrimination data set, loudness, and pitch, to a linear function, a secondorder polynomial, and a third-order polynomial using a least squares criterion. The proportions of variance accounted for by the linear function were .88 and .83 for loudness and pitch, respectively; by the second-order polynomial, they were .96 and .98 , respectively; and by the third-order polynomial, they were .97 and .99 , respectively. The crossover points corresponded to a $57-\mathrm{Hz}$ difference for the lines (376-msec discrimination RT), a $48-\mathrm{Hz}$ difference for the second-order polynomials (372msec RT), and a $47-\mathrm{Hz}$ difference for the third-order polynomials (371-msec RT).

\section{Discussion}

In Experiment 1, we introduced one technique for achieving equal discriminability between dimensions: We tested for the crossover between increasing stimulus differences along one dimension (here, pitch) at a constant stimulus difference along the other dimension (here, loudness). The results indicated that a $10-\mathrm{dB}$ difference in intensity (loudness) corresponds psychologically to an approximately $50-\mathrm{Hz}$ difference in frequency (pitch). Interestingly, these stimulus differences are exactly the ones used by Melara and Marks (1990b) in their study of auditory interaction, which they obtained from extensive pilot testing. It seems likely, therefore, that these stimulus parameters are robust across perceivers, at least with respect to our college-aged subjects. In the remainder of this study, we used the $50-\mathrm{Hz}$ frequency difference as the reference point for all manipulations of the irrelevant dimension.

As mentioned above, because loudness discrimination slowed as the base frequency increased-indicating perhaps another (and as yet unknown) kind of context effectthe pitch matching point was strictly speaking not monolithic, but depended on which loudness task was tested. In particular, loudness discrimination at higher frequencies matched pitch differences smaller than $50 \mathrm{~Hz}$. Still, most of the loudness tasks were accommodated by the $50-\mathrm{Hz}$ point, and otherwise the matching point did not drop below approximately $30 \mathrm{~Hz}$. Across the present set of experiments, we made certain to cover this range of possible matching points. Therefore, the fact that matched discriminability may not correspond exactly to a single value, although intriguing theoretically, had little practical consequence for.our tests.

\section{EXPERIMENT 2 Quantity of Pitches}

In the typical filtering task, the stimulus set contains 4 stimuli: two levels of Dimension 1 at each of two levels of Dimension 2. One can imagine these stimuli, when constituent dimensions are matched in discriminability, as creating a square in psychological space, with each stimulus point residing at one corner of the square. ${ }^{1}$ Suppose in this space that the abscissa corresponds to loudness, the ordinate to pitch. A filtering task along the loudness dimension thus requires subjects to group together stimulus points corresponding to the left side of the square-the soft sounds-to one response key, and points corresponding to the right side of the square-the loud sounds-to the other response key. A baseline task requires subjects to distinguish between, say, the top two points of the square.

Imagine now that we increase the size of the filtering stimulus set by adding points to the left and right sides of the square. This is accomplished by increasing the quantity of frequency values, while maintaining only two values of intensity. A pictorial example is given in the top panel of Figure 2, with stimulus quantity increasing from left to right. From the point of view of response requirements, the tasks are identical: In each case, subjects must group stimulus points falling along the left column apart from points falling along the right column. Nonetheless, behaviorally speaking, grouping in each of the large stimulus sets (on the right side of the panel) may not be the same as grouping in the usual four-stimulus set (on the far left side). We must inquire, therefore, about the possible effects of stimulus quantity on Garner interference. We can pose the same question with respect to linear congruity (the details of which are described below), and we can then compare the pattern of Garner interference with the pattern of linear congruity. These were the goals of Experiment 2.

\section{Method}

Subjects. Forty-two subjects ( 23 men, 19 women) participated in partial fulfillment of a course requirement. None had participated in Experiment 1.

Stimuli, Apparatus, and Procedure. The apparatus was identical to that in Experiment 1. The total stimulus set comprised 102 stimuli, 51 stimuli at each of two intensities $(60$ and $70 \mathrm{~dB})$, ranging in frequency from 900 to $950 \mathrm{~Hz}$ in 1-Hz steps. From this larger set, seven smaller stimulus sets defined seven loudness tasks: one baseline task and six filtering tasks.

Baseline. The frequency was held constant at $900 \mathrm{~Hz},{ }^{2}$ and the subjects discriminated two stimuli-that is, the two values of intensity, soft $(60 \mathrm{~dB})$ versus loud $(70 \mathrm{~dB})$. Each stimulus appeared 100 times during the task (i.e., there were 200 trials total).

2-pitch condition. This was the typical four-stimulus filtering task: the subjects classified soft versus loud, while the irrelevant frequency was either 900 or $950 \mathrm{~Hz}$ on any trial. Each stimulus appeared 50 times during the task (i.e., there were 200 trials total).

3-pitch condition. This was an expanded filtering task containing six stimuli-namely, each of the two values of intensity at 900 , 925 , and $950 \mathrm{~Hz}$. Each stimulus appeared 33 times during the task (i.e., there were 198 trials total).

5-pitch condition. This expanded set contained 10 stimuli, each of the two intensities at 902, 914, 926, 938, and $950 \mathrm{~Hz}$. Each stimulus appeared 20 times during the task (i.e., there were 200 trials total).

10-pitch condition. This set contained 20 stimuli, with each of the two intensities at frequencies ranging from 905 to $950 \mathrm{~Hz}$ in steps of $5 \mathrm{~Hz}$. Each stimulus appeared 10 times during the task (i.e., there were 200 trials total).

25-pitch condition. This set contained 50 stimuli, with each of the two intensities at frequencies ranging from 902 to $950 \mathrm{~Hz}$ in 


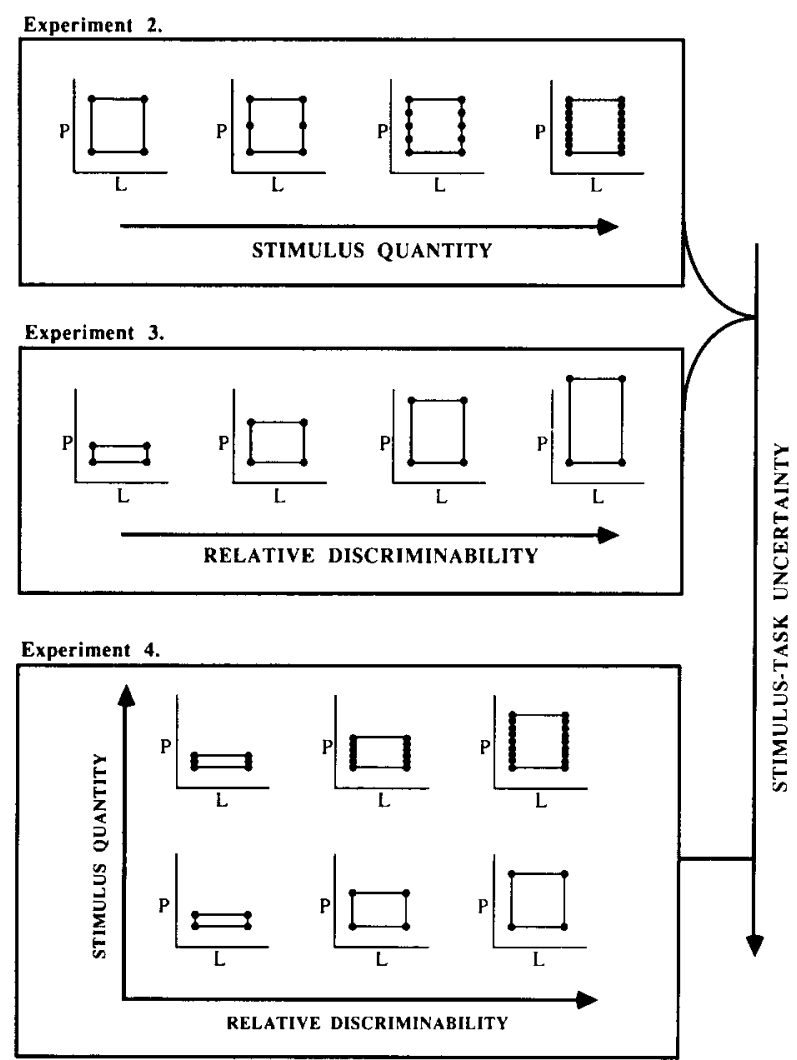

Figure 2. Pictorial examples of the contextual manipulations performed in Experiment 2 (stimulus quantity alone; top panel), Experiment 3 (relative discriminability alone; middle panel) and Experiment 4 (quantity and discriminability combined; bottom panel). Quadrilaterals depict possible representations of each stimulus set in psychological space; filled circles on the quadrilaterals depict the values of pitch (P) and loudness $(L)$ included in each set, with pitch relations varied between sets to create context and loudness values held constant to equate task requirements. Stimulus-task uncertainty is depicted as the change in context from Experiments 2 and 3 , in which quantity and discriminability were varied singly, to Experiment 4 , where quantity and discriminability were varied jointly. Note: These panels are intended only as aids in conceptualizing the context manipulations; they do not define the exact stimulus sets tested in each experiment (see text for specific information on stimulus sets), nor do they represent specific theoretical propositions (see note 1).

steps of $2 \mathrm{~Hz}$. Each stimulus appeared four times during the task (i.e., there were 200 trials total).

50-pitch condition. This set contained 100 stimuli, each of the two intensities at frequencies ranging from 901 to $950 \mathrm{~Hz}$ in steps of $1 \mathrm{~Hz}$. Each stimulus appeared twice during the task (i.e., there were 200 trials total).

The order of presentation of the seven tasks was balanced across subjects by means of a Latin square. The first task for all subjects was performed twice, the first time as practice. Instructions for classifying loudness were presented on the computer screen at the beginning of each task; no mention was made of the quantity of pitches contained in the task. All other aspects of procedure duplicated that of Experiment 1. The experiment lasted approximately $1 \mathrm{~h}$.

\section{Results}

The average error rate in Experiment 2 was 3.12\%. The correlation between speed and accuracy was .94 . Garner interference was computed for each subject by subtracting RT performance at baseline from RT performance in each of the six filtering tasks. Average RT at baseline and in each filtering task are presented graphically in Figure 3.

Garner interference. An ANOVA was performed on Garner interference with stimulus quantity as the only independent variable. The effect was significant $[F(5,205)=$ 3.31, $\left.M S_{\mathrm{e}}=2,817.21, p<01\right]$. Newman-Keuls post hoc analysis (.05 criterion level) indicated that Garner interference in the two-pitch condition was significantly greater than Garner interference in any of the other conditions, which did not differ from one another. An ANOVA of error percentages in each task, and subsequent NewmanKeuls analysis, indicated that two-pitch filtering (3.62\%) was performed significantly worse than baseline $(2.54 \%)$ $\left[F(6,246)=2.60, M S_{\mathrm{e}}=1.74, p<.01\right]$; no other accuracy differences were significant.

What is psychologically most interesting about the RT data, as is apparent from Figure 3, is the precipitous drop in Garner interference (i.e., RT distance from baseline) as the filtering task is expanded beyond the usual fourstimulus case. With two pitches (i.e., four stimuli), subjects suffered $65 \mathrm{msec}$ of interference on the average; with three pitches (i.e., six stimuli), interference fell to $36 \mathrm{msec}$, reaching a stable level of approximately $30 \mathrm{msec}$ as more pitches were added.

Linear congruity. To compute effects of congruity, one typically subtracts the average RT on trials in which stimulus attributes are congruent (e.g., loud/high-pitched or soft/low-pitched) from the average RT on trials in which stimulus attributes are incongruent (e.g., loud/low-pitched or soft/high-pitched). This traditional approach was not viable here, however: Because we used multiple values along the irrelevant dimension, congruity occurred by degrees; thus, there was ambiguity in identifying any

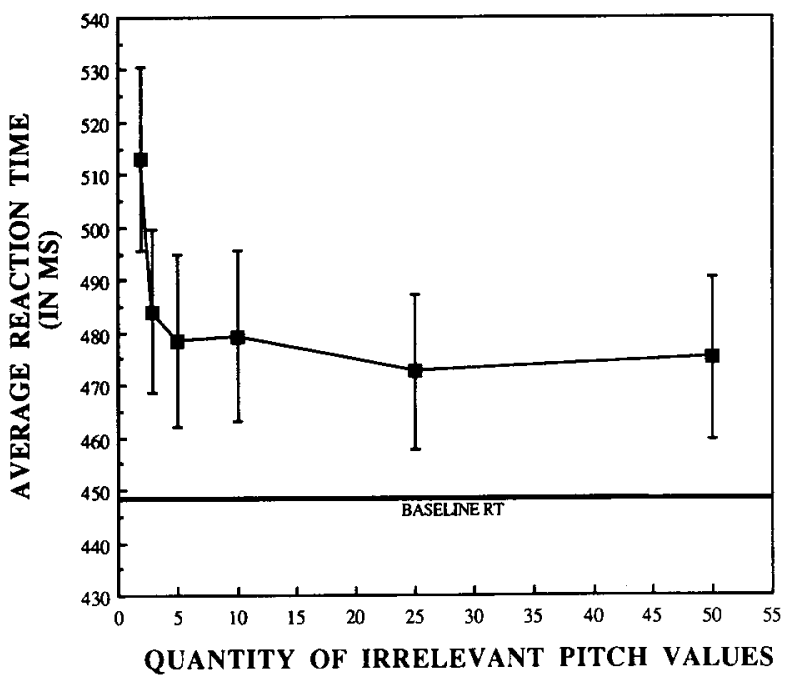

Figure 3. Average reaction time (RT, in milliseconds) in each of the six filtering tasks of Experiment 2. Note: Variability in each condition is shown by standard error bars. The bold horizontal line represents average $\mathrm{RT}$ performance at baseline. 
given trial as congruent or incongruent. We introduce now a new approach called linear congruity.

A subject's RTs to the various stimuli in each response category create two performance distributions in each condition, one for the "soft" category $(60 \mathrm{~dB})$ and one for the "loud" category (70 dB). Performance distributions for a hypothetical subject in the 25-pitch condition appear in Figure 4. Computation begins by determining, for each subject, the linear slope and intercept of each performance distribution in each condition, with RT as the dependent variable and auditory frequency as the independent variable. From these regression equations we derive two predicted RTs in each response category, at frequencies corresponding to end points of the stimulus set used in each task; for all tasks of Experiment 2, these end points were 900 and $950 \mathrm{~Hz}$. We label the predicted RTs for $900 \mathrm{~Hz} /$ soft and $950 \mathrm{~Hz} /$ loud as congruent $\left(\hat{\mathrm{C}}_{1}\right.$ and $\hat{\mathrm{C}}_{2}$, respectively) and the predicted RTs for $900 \mathrm{~Hz} /$ loud and $950 \mathrm{~Hz} /$ soft as incongruent $\left(\hat{I}_{1}\right.$ and $\hat{\mathrm{I}}_{2}$, respectively). These predicted RTs summarize congruity relations across all the stimulus values tested. We define a linear congruity effect (LCE) as the difference between average congruent $\mathrm{RT}$ and average incongruent $\mathrm{RT}$,

$$
\mathrm{LCE}=\left[\left(\hat{\mathrm{I}}_{1}+\hat{\mathrm{I}}_{2}\right)-\left(\hat{\mathrm{C}}_{1}+\hat{\mathrm{C}}_{2}\right)\right] / 2,
$$

a positive value indicating that subjects were faster to respond to the congruent stimuli.

$\hat{\mathrm{C}}_{1}, \hat{\mathrm{C}}_{2}, \hat{\mathrm{I}}_{1}, \hat{\mathrm{I}}_{2}$, and LCE in each condition are shown in Table 1 . From the table it appears that linear congruity decreases and then increases as the number of irrelevant pitches increases. This trend did not materialize statis-

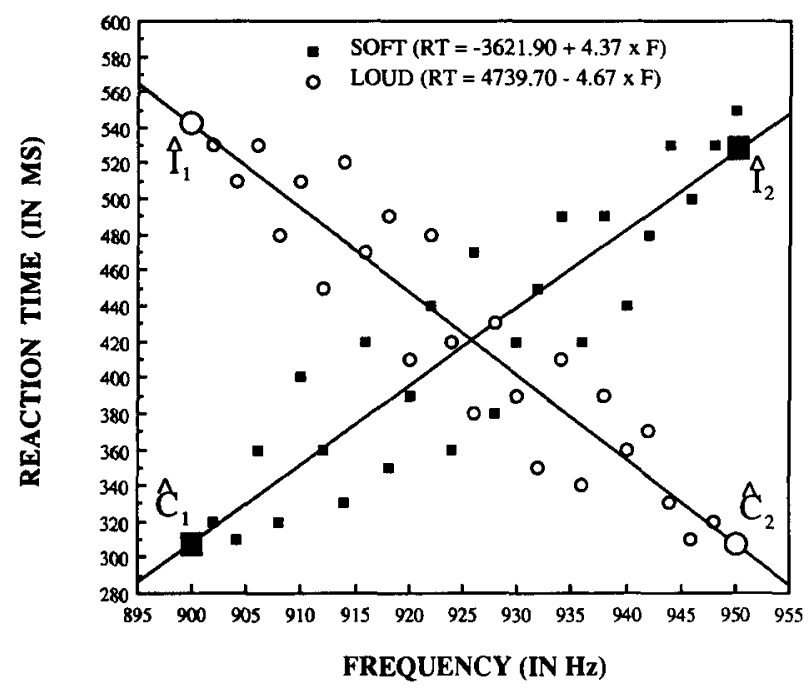

Figure 4. Hypothetical data from loudness classifications in a 25pitch condition. Reaction times (RTs) from each response category (soft, loud) were fit with a least-squares regression line. Predicted RTs for two congruent stimuli- $\hat{C}_{1}$ (for the $900-\mathrm{Hz} /$ soft stimulus) and $\hat{\mathrm{C}}_{2}$ (for the 950-Hz/loud stimulus) - and for two incongruent stimuli$\hat{\mathbf{I}}_{\mathbf{1}}$ (for the 900-Hz/loud stimulus) and $\hat{\mathbf{I}}_{\mathbf{2}}$ (for the 950-Hz/soft stimulus)-are used to derive linear congruity effects (LCE; see Equation 1). In this example, LCE $=+226$ msec.
Table 1

Average Reaction Time (RT, in Milliseconds) to Congruent and Incongruent Stimuli and Linear Congruity Effects (LCEs) in Each of Six Filtering Tasks in Experiment 2

\begin{tabular}{rcccccr}
\hline & \multicolumn{4}{c}{ Tone } & \\
\cline { 2 - 3 } \cline { 5 - 6 } Condition & \multicolumn{2}{c}{ Soft } & \multicolumn{2}{c}{ Loud } & \\
\cline { 2 - 3 } \cline { 5 - 6 } & $900 \mathrm{~Hz}(\mathrm{C})$ & $950 \mathrm{~Hz}(\mathrm{I})$ & & $900 \mathrm{~Hz}(\mathrm{I})$ & $950 \mathrm{~Hz}(\mathrm{C})$ & LCE \\
\hline 2-pitch & 504 & 532 & & 516 & 494 & 25 \\
3-pitch & 478 & 492 & & 497 & 482 & 15 \\
5-pitch & 482 & 498 & & 494 & 478 & 16 \\
10-pitch & 476 & 491 & & 479 & 477 & 8 \\
25-pitch & 467 & 485 & & 493 & 460 & 24 \\
50-pitch & 464 & 494 & & 483 & 467 & 23 \\
\hline
\end{tabular}

Note-The six filtering tasks are specified by the quantity of different pitches contained in the task. Stimulus RTs were estimated from linear regression analyses of response classification functions (see text for details). LCE was computed by subtracting the average RT to congruent stimuli (low-pitched soft tones and high-pitched loud tones) from the average RT to incongruent stimuli (high-pitched soft and low-pitched loud tones). $\mathrm{C}$ and I denote congruent and incongruent stimuli, respectively.

tically, however: the difference among congruity effects across conditions did not approach significance $[F(5,205)$ $=1.01, M S_{\mathrm{e}}=1,910.45$, n.s.]. The important point is that stimulus quantity appears to have had a qualitatively different effect on linear congruity than on Garner interference.

\section{Discussion}

Increases in the quantity of values presented along the irrelevant dimension caused a substantial decrease in Garner interference, but not in linear congruity. Garner interference was maximal when the stimulus set included only two values along the irrelevant dimension. Tasks that included any additional irrelevant values caused Garner interference to decline by half. Interestingly, Garner interference was significantly greater than zero even when the interval of $50 \mathrm{~Hz}$ was filled with 50 different pitches.

The subjects displayed appreciable effects of linear congruity in almost every task tested in Experiment 2: they were faster to respond "loud" to high-pitched sounds, and faster to respond "soft" to low-pitched sounds. These results mimic the results reported by Melara and Marks (1990b) for simple congruity effects with auditory dimensions. In general, a linear relationship captured well the change in RT performance with increases in stimulus frequency. Of course, by definition a line provides a perfect fit when each response category is limited to only two stimuli; thus, the linear model becomes progressively better as the filtering task approximates the typical fourstimulus situation, that being the basis of simple congruity effects. Even so, the present results suggest that simple congruity effects-reconceptualized here as the linear congruity effects that obtain with four stimuli-extend readily to more complicated stimulus sets, serving psychologically, and not merely mathematically, as a special case of linear congruity.

The quantity of irrelevant pitch values had a much greater influence on Garner interference than on linear 
congruity. This implies that the two measures are not surrogates, and that our contextual manipulation might serve as a useful approach for dissociating them. What psychological mechanisms cause the two different patterns of performance is not obvious from the present experiment. In this study, we will focus on whether the dissociating effects of stimulus quantity are consistent across experiments, and whether other contextual manipulations also dissociate these dependent variables. Once all of these parts are assembled, we can reach general conclusions about the varied effects of context on these two indices of dimensional interaction. We defer further discussion until then.

\section{EXPERIMENT 3 Discriminability of Pitches}

In Experiment 3, each filtering set contained only four stimulus points, and we manipulated the shape of the quadrilateral that these points formed. In particular, we varied the distance between points along the ordinate (pitch), thereby testing classification performance in response to the presentation of differently shaped rectangles. This was accomplished by manipulating the relative discriminability of pitch, the irrelevant dimension, while leaving the discriminability of loudness constant at $10 \mathrm{~dB}$. Thus, for filtering tasks involving pitch variation of less than $50 \mathrm{~Hz}-$ that being the perceptual matching point for a $10-\mathrm{dB}$ difference in intensity-subjects classified points on short, fat rectangles in psychological space. For filtering tasks in which the pitch difference was greater than $50 \mathrm{~Hz}$, subjects faced tall, thin psychological rectangles. The center panel of Figure 2 captures these ideas pictorially. We asked how changes in discriminability would affect Garner interference and linear congruity.

\section{Method}

Subjects. Forty-four undergraduates ( 22 men and 22 women) participated in partial fulfillment of a course requirement. None were subjects in any of the earlier experiments.

Stimuli, Apparatus, and Procedure. The apparatus was identical to that in the earlier experiments. Each subject classified sounds as "soft" $(60 \mathrm{~dB})$ or "loud" $(70 \mathrm{~dB})$ in 1 baseline task and 10 filtering tasks. Each task contained 128 trials. In the baseline task, the frequency was held constant at $900 \mathrm{~Hz}$. Each filtering task contained four stimuli, two levels of frequency at each level of intensity. The filtering tasks were distinguished by the frequency difference, from 10 to $100 \mathrm{~Hz}$ in steps of $10 \mathrm{~Hz}$, with the lower frequency always at $900 \mathrm{~Hz}$. Order of task presentation was specified by a Latin square, with the first task in the sequence serving as practice, as in earlier experiments. All other aspects duplicated those of the earlier experiments. The experiment lasted approximately $75 \mathrm{~min}$.

\section{Results}

Overall performance. The subjects committed 3.35\% errors on the average. Speed correlated .91 with accuracy. Accuracy differed significantly across tasks $[F(10,430)$ $\left.=12.71, M S_{\mathrm{e}}=2.37, p<.001\right]$. In general, subjects committed more errors in judging loudness the greater the difference in frequency. At baseline, errors averaged $2.27 \%$; a $10-\mathrm{Hz}$ frequency difference led to $2.20 \%$ errors, a $50-\mathrm{Hz}$ difference to $3.60 \%$ errors, and a $100-\mathrm{Hz}$ difference to $4.63 \%$ errors.

Garner interference. Average RT at baseline and in each filtering task are depicted in Figure 5. Garner interference was subjected to ANOVA with task (i.e., frequency difference; 10 levels) as the only factor; the effect of task was significant $\left[F(9,387)=8.25, M S_{\mathrm{e}}=\right.$ $2,608.27, p<001]$. As can be seen in Figure 5, and as confirmed by Newman-Keuls post hoc analysis (.05 level), the magnitude of Garner interference was significantly greater at the larger versus the smaller frequency differences. In the $50-\mathrm{Hz}$ condition, in which dimensions were presumably matched in discriminability, Garner interference averaged $52 \mathrm{msec}$, slightly less than the $65 \mathrm{msec}$ found in the same condition in Experiment 2. The magnitude of Garner interference was smaller in the $10-\mathrm{Hz}$ condition $(31 \mathrm{msec})$ and larger in the $100-\mathrm{Hz}$ condition (104 msec).

Linear congruity. LCEs and average RTs to congruent and incongruent stimuli in each condition appear in Table 2 . An ANOVA of congruity effects revealed a significant effect of task $\left[F(9,387)=20.99, M S_{\mathrm{e}}=1,056.20\right.$, $p<.001]$. Increases in discriminability caused a regular rise in the magnitude of LCE. Newman-Keuls analysis showed, for example, that the average congruity effect at $100 \mathrm{~Hz}(67 \mathrm{msec})$ was significantly larger than that in all but the $90-\mathrm{Hz}$ condition; on the other hand, the average linear congruity at $10 \mathrm{~Hz}(-3 \mathrm{msec})$ was significantly smaller than that in all but the $20-\mathrm{Hz}$ condition.

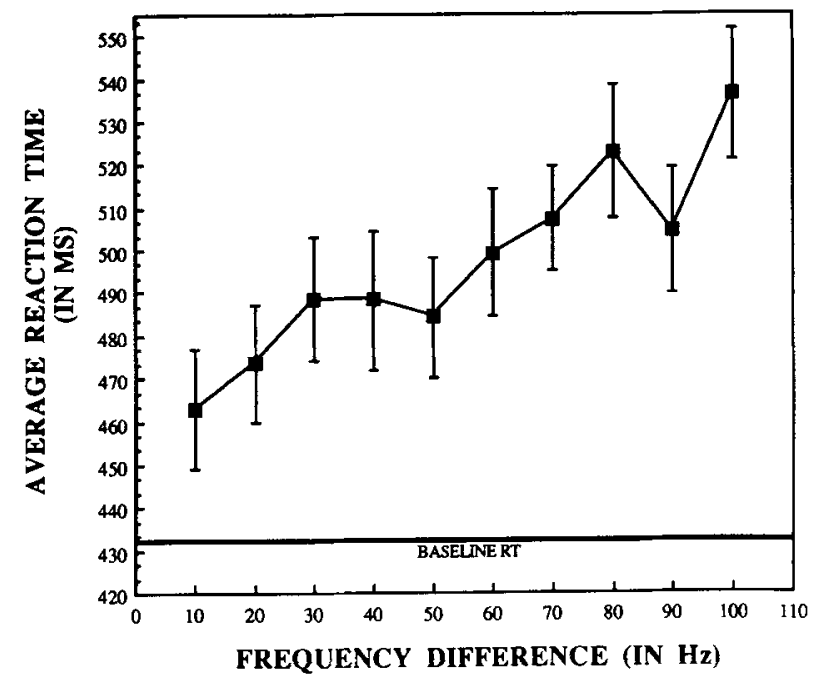

Figure 5. Average reaction time (RT, in milliseconds) in each of the 10 filtering tasks of Experiment 3. The 10 filtering tasks are specified by the difference in auditory frequency (in hertz) of values along the irrelevant dimension (i.e., pitch): 10, 20, 30, 40, 50, 60, 70, 80, 90 , and 100 Hz. Note: Variability in each condition is shown by standard error bars. The bold horizontal line represents average RT performance at baseline. 
Table 2

Average Reaction Time (RT, in Milliseconds) to Congruent and Incongruent Stimuli and Linear Congruity Effects (LCEs) in Each of the 10 Filtering Tasks in Experiment 3

\begin{tabular}{|c|c|c|c|c|c|}
\hline \multirow[b]{3}{*}{ Condition } & \multicolumn{4}{|c|}{ Tone } & \multirow[b]{3}{*}{ LCE } \\
\hline & \multicolumn{2}{|c|}{ Soft } & \multicolumn{2}{|c|}{ Loud } & \\
\hline & Low (C) & High (I) & Low (I) & High (C) & \\
\hline $10-\mathrm{Hz}$ & 477 & 466 & 458 & 452 & -3 \\
\hline $20-\mathrm{Hz}$ & 479 & 478 & 473 & 465 & 4 \\
\hline $30-\mathrm{Hz}$ & 487 & 504 & 490 & 474 & 16 \\
\hline $40-\mathrm{Hz}$ & 491 & 505 & 493 & 465 & 21 \\
\hline $50-\mathrm{Hz}$ & 480 & 507 & 486 & 464 & 30 \\
\hline $60-\mathrm{Hz}$ & 488 & 528 & 497 & 475 & 31 \\
\hline $70-\mathrm{Hz}$ & 502 & 520 & 525 & 471 & 36 \\
\hline $80-\mathrm{Hz}$ & 512 & 549 & 535 & 493 & 40 \\
\hline $90-\mathrm{Hz}$ & 487 & 536 & 532 & 464 & 60 \\
\hline $100-\mathrm{Hz}$ & 518 & 570 & 570 & 487 & 68 \\
\hline
\end{tabular}

Note-The 10 filtering tasks are specified by the difference in auditory frequency (in $\mathrm{Hz}$ ) of low versus high values along the pitch dimension. Stimulus RTs are averages from observed data. The columns labeled Low represent stimuli with a frequency of $900 \mathrm{~Hz}$. The columns labeled High represent stimuli whose frequency is the sum of $900 \mathrm{~Hz}$ and the frequency of the particular condition (e.g., High in the $60-\mathrm{Hz}$ condition is $960 \mathrm{~Hz}$ ). LCE was computed by subtracting the average RT to congruent stimuli (low-pitched soft tones and high-pitched loud tones) from the average RT to incongruent stimuli (high-pitched soft and lowpitched loud tones). $\mathrm{C}$ and I denote congruent and incongruent stimuli, respectively.

\section{Discussion}

Experiment 3 showed that increases in discriminability of the irrelevant dimension have a monotonically increasing effect on the magnitude of both Garner interference and linear congruity in loudness classification. Increasing the range of irrelevant variation progressively worsened the subjects' ability to attend selectively to the relevant dimension. In geometric terms, we can say that selective attention failure varies lawfully around a point of equal discriminability - that is, the perceptual square created by differences of $50 \mathrm{~Hz}$ and $10 \mathrm{~dB}$ : interactive effects are small for short, fat rectangles and large for tall, thin rectangles (see Figure 2). Unlike stimulus quantity, discriminability affects Garner interference and linear congruity in similar fashion.

\section{EXPERIMENT 4 Quantity and Discriminability Below the Matching Point}

In the preceding experiments, only a single stimulus variable was manipulated at a time-either discriminability (Experiments 1 and 3) or stimulus quantity (Experiment 2). In the remaining experiments of this study, we evaluated how combinations of these two stimulus contexts might influence dimensional interaction. In Experiment 4, three levels of discriminability were combined with two levels of stimulus quantity, creating six filtering tasks. The discriminabilities tested $-10,25$, and $50 \mathrm{~Hz}$-were all at or below the matching point. For each of these discriminabilities, the stimulus quantity was either two or else the number of integer frequencies in the range.
As before, one can conceptualize these tasks in spatial terms: If the stimulus sets created by each of the three discriminabilities are each represented by a rectangle in psychological space, the left and right sides of the rectangle were either unfilled (i.e., composed of only 2 stimulus points) or filled (i.e., composed of 10,25 , or 50 stimulus points). The bottom panel of Figure 2 provides a graphical representation. As discussed earlier, by manipulating within a single experimental session both of the lower order contextual variables-quantity and discriminability-we effectively increase the stimulus-task uncertainty of the session, relative to that of the earlier experiments. In Figure 2, each panel (i.e., experiment) corresponds to a value along an uncertainty axis; Experiment 4 entails greater uncertainty than either Experiment 2 or Experiment 3. Accordingly, we expected more pronounced effects of interactive processing in Experiment 4, as measured by interference and congruity, relative to the earlier, lowuncertainty situations. Nonetheless, we were surprised to find that uncertainty exhibited an unusually powerful effect on classification.

\section{Method}

Subjects. Twenty-one Purdue undergraduates (8 men and 13 women) participated in this experiment in partial fulfillment of a course requirement. None had participated in any of the earlier experiments.

Stimuli, Apparatus, and Procedure. Stimuli were 102 sine-wave signals, ranging in frequency from 900 to $950 \mathrm{~Hz}$ in $1-\mathrm{Hz}$ steps, and presented at either 60 or $70 \mathrm{~dB}(\mathrm{~A})$. This set was used to create seven loudness tasks of 200 trials each-namely, one baseline task and six filtering tasks. The baseline task was identical to those used in Experiments 2 and 3. The filtering tasks were labeled and defined as follows.

$10-H z$ unfilled. This task contained four stimuli, the two values of intensity presented at frequencies of 921 or $930 \mathrm{~Hz}$. Each stimulus was presented 50 times. The task was analogous to the $10-\mathrm{Hz}$ task used in Experiment 3.

25- $\mathrm{Hz}$ unfilled. A four-stimulus task (i.e., 50 presentations of each stimulus), with frequencies of 913 and $937 \mathrm{~Hz}$, this task was analogous to the 25- $\mathrm{Hz}$ task used in Experiment 3.

$50-\mathrm{Hz}$ unfilled. Frequencies here were 901 and $950 \mathrm{~Hz}$, creating four stimuli (i.e., 50 presentations of each stimulus). The task was analogous to the two-pitch task used in Experiment 2 and the $50-\mathrm{Hz}$ task used in Experiment 3.

$10-\mathrm{Hz}$ filled. This task contained 20 stimuli, each presented 10 times, with frequencies ranging from 921 to $930 \mathrm{~Hz}$ in $1-\mathrm{Hz}$ steps.

25-Hz filled. This task contained 50 stimuli, each presented four times, with frequencies ranging from 913 to $937 \mathrm{~Hz}$ in 1-Hz steps.

50- $\mathrm{Hz}$ filled. This task contained 100 stimuli, each presented twice, with frequencies ranging from 901 to $950 \mathrm{~Hz}$ in $1-\mathrm{Hz}$ steps. The task was analogous to the 50-Pitch task used in Experiment 2.

All aspects of stimulus presentation, apparatus, and procedure were similar to those in the previous experiments. The experiment lasted approximately $1 \mathrm{~h}$.

\section{Results}

Overall performance. The subjects made $4.20 \%$ errors of classification on the average. The correlation between speed and accuracy was .08. An ANOVA of error percentages revealed a reliable effect of task $[F(6,120)=$ $\left.15.68, M S_{\mathrm{e}}=2.29, p<.001\right]$. The subjects were significantly more error prone in the $10-\mathrm{Hz}$ unfilled task 
(6.93\%) than in any of the other tasks, which did not differ from one another. The relatively poor accuracy in the 10$\mathrm{Hz}$ unfilled task may represent a slight tendency to trade off speed for accuracy, since classifications in this task were among the fastest. The causes of this tradeoff are unknown.

Garner interference. Figure 6 depicts average RT at baseline and in filled and unfilled tasks at each of the three frequency differences. An ANOVA was performed on Garner interference with frequency difference (three levels: 10,25 , and $50 \mathrm{~Hz}$ ) and stimulus quantity (two levels: filled and unfilled) as factors. There was a significant effect of frequency difference $\left[F(2,40)=14.16, M S_{\mathrm{e}}=1,691.95\right.$, $p<.001$ ]: Garner interference in the $50-\mathrm{Hz}$ range $(55 \mathrm{msec})$ was significantly greater than interference in the $25-\mathrm{Hz}$ range $(29 \mathrm{msec})$ which, in turn, was greater than interference in the $10-\mathrm{Hz}$ range $(7 \mathrm{msec})$. There was also a trend toward greater interference in the unfilled condition $(43 \mathrm{msec})$ than in the filled condition $(18 \mathrm{msec})$ $\left[F(1,20)=3.71, M S_{\mathrm{e}}=5,513.18, p=.06\right]$. The interaction between factors was not significant $[F(2,40)=$ $2.01, M S_{c}=2,207.54$, n.s.].

Linear congruity. Table 3 contains congruent and incongruent RTs and LCEs in each filtering task. It is immediately obvious from the table that the pattern of linear congruity has changed astonishingly from earlier experiments. Indeed, congruity effects are opposite in direction to those obtained earlier: At lower frequencies, the subjects were generally faster to respond "loud" than "soft"; at higher frequencies, the subjects were generally faster to respond "soft" than "loud." In each condition, therefore, linear congruity was negative.

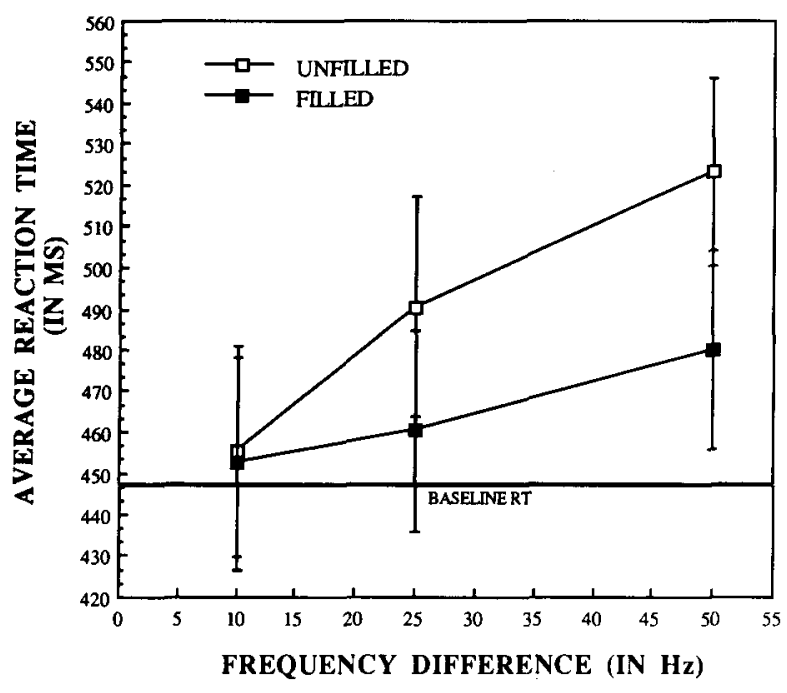

Figure 6. Average reaction time (RT, in milliseconds) in each of the six filtering tasks of Experiment 4 . The parameter specifies stimulus quantity (filled, unfilled) and the abscissa specifies the difference in auditory frequency (in hertz) of values along the irrelevant dimension $(10,25$, and $50 \mathrm{~Hz})$. Note: Variability in each condition is shown by standard error bars. The bold horizontal line represents average $\mathrm{RT}$ performance at baseline.
Table 3

Average Reaction Time (RT, in Milliseconds) to Congruent and Incongruent Stimuli and Linear Congruity Effects (LCEs) in Each of Six Filtering Tasks in Experiment 4

\begin{tabular}{lcccccr} 
& \multicolumn{4}{c}{ Tone } & \\
\cline { 2 - 3 } \multicolumn{1}{c}{ Condition } & \multicolumn{2}{c}{ Soft } & & \multicolumn{2}{c}{ Loud } & \\
\cline { 2 - 3 } \cline { 5 - 6 } & Low (C) & High (I) & & Low (I) & High (C) & LCE \\
\hline $10-\mathrm{Hz}$ unfilled & 463 & 461 & & 443 & 455 & -7 \\
10-Hz filled & 465 & 467 & & 431 & 447 & -7 \\
$25-\mathrm{Hz}$ unfilled & 509 & 505 & & 470 & 497 & -16 \\
$25-\mathrm{Hz}$ filled & 475 & 465 & & 425 & 468 & -27 \\
$50-\mathrm{Hz}$ unfilled & 549 & 510 & & 477 & 556 & -59 \\
$50-\mathrm{Hz}$ filled & $\mathbf{5 0 3}$ & 460 & & 438 & 516 & -61 \\
\hline
\end{tabular}

Note-Stimulus RTs were estimated from linear regression analyses of response classification functions (see text for details). The labels Low and High correspond to the stimuli with the lowest frequency and the highest frequency, respectively, from the range of stimuli tested in a particular condition (see text for specific stimulus values). LCE was computed by subtracting the average RT to congruent stimuli (low-pitched soft tones and high-pitched loud tones) from the average RT to incongruent stimuli (high-pitched soft and low-pitched loud tones). C and I denote congruent and incongruent stimuli, respectively.

An ANOVA was performed on congruity effects, with frequency difference and stimulus quantity as factors. Only frequency difference led to a significant effect $\left[F(2,40)=34.77, M S_{\mathrm{e}}=893.75, p<.001\right]$. Linear congruity at $50 \mathrm{~Hz}(-60 \mathrm{msec})$ was significantly more negative than congruity at $25 \mathrm{~Hz}(-22 \mathrm{msec})$, which, in turn, was significantly more negative than congruity at $10 \mathrm{~Hz}(-7 \mathrm{msec})$, as tested by Newman-Keuls analysis.

\section{Discussion}

Combining in a single experiment variations in relative discriminability with variations in stimulus quantity produced patterns of interactive processing markedly different from those obtained when these factors were varied alone. Particularly noteworthy was the extraordinary reversal of direction in linear congruity: In Experiment 3, increases in discriminability were accompanied by increasingly positive congruity effects; in this experiment, increases in discriminability were accompanied by increasingly negative congruity effects.

Uncertainty also steepened the rate at which linear congruity increased. This can be seen by considering the slopes of the best-fitting lines that relate discriminability to congruity. In Experiment 3, the slope was .71 ( $r^{2}=$ $.96)$; in the present experiment, the slope had increased in absolute value to 1.35 (filled; $r^{2}=.95$ ) and 1.33 (unfilled; $r^{2}=1.00$ ). The upshot is that the exact same task produced very different magnitudes of linear congruity from experiment to experiment. For example, at the point of equal discriminability $(50 \mathrm{~Hz})$, the average absolute value of linear congruity was $60 \mathrm{msec}$ in Experiment 4 (unfilled $=59 \mathrm{msec}$; filled $=61 \mathrm{msec}$ ) but was only $25 \mathrm{msec}$ in Experiment 3, and only $24 \mathrm{msec}$ in Experiment 2 (2-pitch $=24 \mathrm{msec} ; 50$-pitch $=23 \mathrm{msec}$ ).

Patterns of Garner interference in Experiment 4 also differed from what previous experiments had led us to expect, although the deviations here were somewhat less dramatic than those observed in linear congruity. Con- 
sider a linear function that relates discriminability to Garner interference. In Experiment 3, the slope of this function was $.68\left(r^{2}=.87\right)$; in the present experiment, the corresponding (i.e., unfilled) slope jumped to 1.66 $\left(r^{2}=.97\right)$. At the same time, there was a substantial decrease in the intercept of the Garner interference function: from 27.00 in Experiment 3 to -3.92 in Experiment 4. In terms of magnitudes, Experiment 3 saw a change in Garner interference from 10 to $50 \mathrm{~Hz}$ of only $21 \mathrm{msec}$ (i.e., from 31 to $52 \mathrm{msec}$ ). In Experiment 4, however, the range of Garner interference in the unfilled condition was $67 \mathrm{msec}$ (i.e., from 9 to $76 \mathrm{msec}$ ).

Also of interest was the relatively weak effect on classification of stimulus quantity. From the results of Experiment 2, we anticipated a precipitous drop in Garner interference as the sides of the stimulus rectangle filled with points. Admittedly, Garner interference was numerically smaller in the filled than in the unfilled condition. Nonetheless, in statistical terms, this decrease in magnitude was uneventful.

Role of uncertainty. What accounts for the powerful effects of uncertainty on the form and magnitude of interactive processing? In our view, uncertainty causes response adjustment, analogous to a shift in adaptation level (Helson, 1964). Such adjustment implies a commensurate change in classification success, to accommodate the set of tasks contained within an experiment. Accordingly, we expected uncertainty to yield a general shift in performance. But results from Experiment 4 indicate that this is not the case. The evidence suggests instead that uncertainty affects classification by interacting statistically with stimulus discriminability: It modifies both the slope and the intercept of Garner interference functions. Psychologically, this means that uncertainty blurs the effects of discriminability at low levels of frequency difference (e.g., the 10- $\mathrm{Hz}$ task), but heightens the effects of discriminability at high levels of frequency difference (e.g., the $50-\mathrm{Hz}$ task). In the remaining experiments of this study, we evaluated several expectations that emerged from the idea that uncertainty mediates the effects of discriminability.

Reversal in linear congruity. It is difficult to know what accounts for the surprising change in linear congruity seen in this experiment. We might suppose, as has been suggested previously (Melara \& Marks, 1990b), that effects of simple congruity with auditory stimuli reflect a special status that observers accord stimuli whose constituent attributes are congruent in sign. In unfilled tasks, for example, the values "loud" and "high-pitched" both derive from the positive poles of their respective continua. Congruent or incongruent attribute signs are necessarily relative: $925 \mathrm{~Hz}$ is high pitched when paired with $900 \mathrm{~Hz}$ but low pitched when paired with $950 \mathrm{~Hz}$. If we assume that sign coding does indeed occur, the results from Experiment 2 indicate that signs are applied continuously across a dimension, because the relative frequency of stimuli served as a useful predictor of RT across the stimulus range in those filled tasks. Given these arguments, one might hazard that increases in stimulus uncertainty in pitch, as occurred in Experiment 4, tended to make rela- tive coding of the irrelevant dimension more difficult; pitch values would need to change sign, depending on the task. Uncertainty would thus be expected to engender a decrease in the magnitude of linear congruity.

Even so, it is unclear why linear congruity actually reversed in Experiment 4. One possibility is that while pitch would tend now to be coded in more absolute terms, loudness, being assigned the same two values in each task, could still be coded relatively. This difference might engender a shift in processing priority, from sign match to sign contrast. The change would thus be analogous to the shift from stimulus assimilation to stimulus contrast seen in psychophysical judgments (Helson, 1964; Ward \& Lockhead, 1971). Despite the merits of this idea, however, many other hypotheses are possible, including the chance that the reversal found in Experiment 4 was anomalous. In the remaining experiments, we measure linear congruity under new high-uncertainty situations and ask whether these situations always promote reverse linear congruity.

\section{EXPERIMENT 5 \\ Quantity and Discriminability at High Uncertainty}

To evaluate the hypothesis that failures of selective attention are caused, in part, by a statistical interaction between stimulus-task uncertainty and relative discriminability, we increased uncertainty in Experiment 5 by adding three new conditions to the task set used in Experiment 4. Specifically, in addition to the filled and unfilled tasks of Experiment 4 we included three constant-fill tasks. The three tasks $-10-\mathrm{Hz}$ constant fill, $25-\mathrm{Hz}$ constant fill, and 50-Hz constant fill-differed in pitch discriminability, but each contained five levels of frequency. Thus the trialto-trial changes in pitch were smaller on the average than in the corresponding unfilled conditions, but larger on the average than in the corresponding filled conditions (see Lockhead, 1992). We predicted, therefore, that the magnitudes of effects in the constant-fill conditions would fall midway between those obtained in the other two conditions. More important, if discriminability and uncertainty interact statistically, we would expect an increase in the slopes, but a decrease in the intercepts, of the functions relating Garner interference to frequency difference, in comparison with the slopes and intercepts for the same conditions (e.g., unfilled) in the earlier experiments. Finally, if increases in stimulus and task uncertainty underlie the prominent reversal in linear congruity found in Experiment 4 , we would expect replication of this reverse effect in Experiment 5.

\section{Method}

Subjects. Thirty Purdue undergraduates (15 men and 15 women) participated in partial fulfillment of a course requirement. None participated in any of the other experiments in this study.

Stimuli, Apparatus, and Procedure. The 7 tasks used in Experiment 4 were included in a series of 10 tasks. The 3 other tasks, called constant-fill tasks, each contained 20 presentations of each of 10 stimuli ( 200 trials total). In the $10-\mathrm{Hz}$ constant-fill task, the 
two levels of intensity $(60$ and $70 \mathrm{~dB})$ were combined with frequencies ranging from 921 to $933 \mathrm{~Hz}$ in steps of $3 \mathrm{~Hz}$. In the $25-\mathrm{Hz}$ constant-fill task, the stimulus set included frequencies ranging from 913 to $937 \mathrm{~Hz}$ in steps of $6 \mathrm{~Hz}$. In the $50-\mathrm{Hz}$ constant-fill task, the stimulus set included frequencies ranging from 901 to $949 \mathrm{~Hz}$ in steps of $12 \mathrm{~Hz}$

Order of presentation of the 10 tasks was determined by Latin square. All other aspects of stimulus presentation, apparatus, and procedure duplicated those of Experiment 4.

\section{Results}

Overall performance. The subjects committed $3.06 \%$ errors on the average. The correlation between speed and accuracy was -.21 , suggesting, again for unknown reasons, a slight tradeoff between these variables. An ANOVA was performed on error percentages in the 10 tasks. The effect of task was significant $\left[F(9,261)=12.82, M S_{\mathrm{e}}=\right.$ $2.22, p<.001]$. Accuracy of performance in the $25-\mathrm{Hz}$ unfilled task ( $3.63 \%$ errors), the $10-\mathrm{Hz}$ filled task (3.85\% errors), and the $10-\mathrm{Hz}$ unfilled task (5.27\% errors) was generally worse than accuracy in the other tasks. As we shall see, RTs in these three tasks were among the fastest.

Garner interference. Average RTs in the baseline and filtering tasks appear in Figure 7. An ANOVA was performed on Garner interference, with frequency difference (3 levels: 10,25 , and $50 \mathrm{~Hz}$ ) and stimulus quantity (3 levels: filled, constant fill, unfilled) as factors. There was a significant effect of frequency difference $[F(2,58)=$ $\left.69.38, M S_{\mathrm{e}}=1,726.60, p<.001\right]$. Newman-Keuls analysis showed that Garner interference at the $50-\mathrm{Hz}$ frequency difference $(78 \mathrm{msec})$ was significantly greater than Garner interference at the $25-\mathrm{Hz}$ frequency difference $(30 \mathrm{msec})$, which, in turn, was significantly greater than

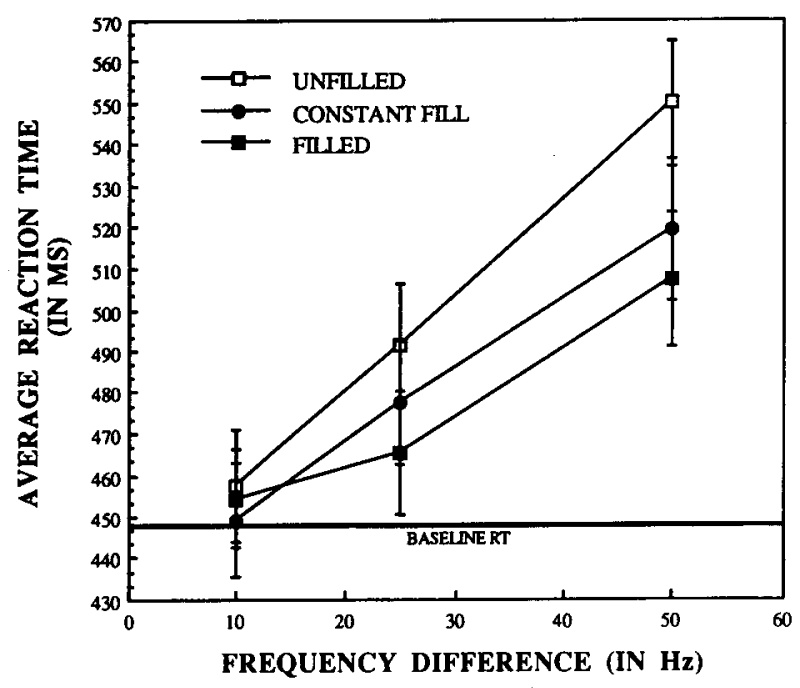

Figure 7. Average reaction time (RT, in milliseconds) in each of the nine filtering tasks of Experiment 5. The parameter specifies stimulus quantity (filled, constant fill, unfilled), and the abscissa specifies the difference in auditory frequency (in hertz) of values along the irrelevant dimension $(10,25$, and $50 \mathrm{~Hz})$. Note: Variability in each condition is shown by standard error bars. The bold horizontal line represents average RT performance at baseline.
Table 4

Average Reaction Time (RT, in Milliseconds) to Congruent and Incongruent Stimuli and Linear Congruity Effects (LCEs) in Each of Nine Filtering Tasks in Experiment 5

\begin{tabular}{lcccccr}
\hline & \multicolumn{5}{c}{ Tone } & \\
\cline { 2 - 3 } \multicolumn{1}{c}{ Condition } & \multicolumn{2}{c}{ Soft } & & \multicolumn{2}{c}{ Loud } & \\
\cline { 2 - 3 } \cline { 5 - 6 } & Low (C) & High (I) & & Low (I) & High (C) & LCE \\
\hline 10-Hz unfilled & 462 & 469 & & 443 & 455 & -3 \\
10-Hz constant & 452 & 462 & & 430 & 450 & -6 \\
10-Hz filled & 456 & 472 & & 440 & 450 & 3 \\
25-Hz unfilled & 498 & 502 & & 465 & 498 & -15 \\
$25-\mathrm{Hz}$ constant & 484 & 481 & & 454 & 488 & -18 \\
$25-\mathrm{Hz}$ filled & 465 & 472 & & 446 & 477 & -12 \\
$50-\mathrm{Hz}$ unfilled & 572 & 531 & & 510 & 586 & -59 \\
$50-\mathrm{Hz}$ constant & 532 & 519 & & 474 & 551 & -45 \\
$50-\mathrm{Hz}$ filled & 522 & 499 & & 459 & 543 & -53 \\
\hline
\end{tabular}

Note-Stimulus RTs were estimated from linear regression analyses of response classification functions (see text for details). The labels Low and High correspond to the stimuli with the lowest frequency and the highest frequency, respectively, from the range of stimuli tested in a particular condition (see text for specific stimulus values). LCE was computed by subtracting the average RT to congruent stimuli (low-pitched soft tones and high-pitched loud tones) from the average RT to incongruent stimuli (high-pitched soft and low-pitched loud tones). C and I denote congruent and incongruent stimuli, respectively.

Garner interference at the $10-\mathrm{Hz}$ frequency difference $(6 \mathrm{msec})$. There was also a significant effect of stimulus quantity $\left[F(2,58)=11.32, M S_{\mathrm{e}}=1,221.60, p<.001\right]$. Performance in unfilled tasks $(52 \mathrm{msec})$ led to significantly more Garner interference than did performance in filled tasks ( $34 \mathrm{msec})$ or in constant-fill tasks $(28 \mathrm{msec})$, which did not differ from each other. There was no interaction between frequency difference and stimulus quantity $\left[F(4,116)=1.58, M S_{\mathrm{e}}=1,990.13\right.$, n.s. $]$.

Linear congruity. The average linear congruity scores in each filtering task appear in Table 4 . It is clear from the table that the strong negative LCEs that obtained in Experiment 4 have been replicated.

An ANOVA of linear congruity scores revealed a significant effect of frequency difference $[F(2,58)=22.21$, $\left.M S_{\mathrm{e}}=2,764.13, p<.001\right]$ but no effect of stimulus quantity $\left[F(2,58)=.59, M S_{\mathrm{e}}=880.09\right.$, n.s. $]$ and no interaction $\left[F(4,116)=1.29, M S_{\mathrm{e}}=741.46, p<.001\right]$. Increases in frequency difference caused the congruity effect to become more negative: Linear congruity was significantly more negative at $50 \mathrm{~Hz}(-52 \mathrm{msec})$ than at $25 \mathrm{~Hz}(-15 \mathrm{msec})$ or $10 \mathrm{~Hz}(-2 \mathrm{msec})$; the latter two conditions did not differ from each other.

\section{Discussion}

Experiment 5 lends support to the hypothesis advanced in Experiment 4: namely, that the differences across experiments in the magnitude of effects are due largely to the mediating influences of stimulus-task uncertainty as it interacts with the discriminability of the irrelevant dimension. Compared with Experiments 3 and 4, Experiment 5 involved an increase in uncertainty because three constant-fill tasks were added to the task set. And, as expected, this increase caused lawful changes in Garner interference relative to the results of Experiments 3 and 4. 
Specifically, uncertainty increased the slope and decreased the intercept of the Garner interference functions. The slope in the unfilled condition, for example, was 2.31 in this experiment $\left(r^{2}=1.00\right)$, compared with 1.66 in the identical condition of Experiment 4, and .68 in Experiment 3 . This change was accompanied by a corresponding decrease in the function's intercept: from 27.00 in Experiment 3 to -3.92 in Experiment 4 to -13.77 in Experiment 5. Similarly, the slope of the Garner interference function in the filled condition $\left(1.35 ; r^{2}=.97\right)$ was almost twice as steep as the slope observed in the identical condition in Experiment $4\left(.70 ; r^{2}=.99\right)$, while the intercept dropped from -2.28 to -10.61 . Obviously, then, experimental context, in the form of stimulus-task uncertainty, is operating powerfully in this study: It masks the effects of small pitch differences, but enhances the effects of large pitch differences.

Unlike in Experiment 4, stimulus quantity produced a reliable change in Garner interference, with interference in the unfilled conditions exceeding that in the other conditions. Again, it is possible that the greater influence of quantity in this experiment was mediated by the change in task context. This idea is difficult to defend, however, because it does not explain why the relatively simple task situation of Experiment 2 led to such strong effects of stimulus quantity. We shall see later that, although stimulus quantity had a consistently greater influence on Garner interference than on linear congruity in this study (as was found in this experiment), it generally enjoyed only a secondary influence on the magnitude of effects relative to the other contextual factors (see Gravetter \& Lockhead, 1973).

Experiment 5 replicated the large negative effects of linear congruity observed in Experiment 4; it is clear that the finding of negative linear congruity is not anomalous. Moreover, the slopes of the linear congruity functions were slightly more negative than those observed in Experiment 4 [filled: -1.36 (Experiment 4 ) vs. -1.42 (Experiment 5; $r^{2}=.98$ ); unfilled: -1.33 (Experiment 4 ) vs. -1.44 (Experiment $5 ; r^{2}=.97$ ) , whereas the intercepts were substantially more positive (filled: $6.66 \mathrm{vs}$. 19.81; unfilled: 10.38 vs. 15.59 ). This combination suggests a statistical interaction of uncertainty and discriminability on linear congruity, a pattern consistent with (albeit in an opposite direction to) that observed in Garner interference. (In the General Discussion, we will report the results of across-experiment regression analyses on Garner interference and linear congruity to test explicitly for these hypothesized interactions.) This set of findingsthat is, (1) a change from positive congruity effects (Experiments 2 and 3 ) to negative congruity effects (Experiments 4 and 5) and (2) a change in both the slopes and intercepts of linear congruity functions across experiments-lends further support to our claim that stimulustask uncertainty causes powerful changes in the ability of human observers to focus on one source of information in their environment.

\section{EXPERIMENT 6 \\ Quantity and Discriminability Above the Matching Point}

When we simultaneously manipulated the two lower order contextual variables in Experiments 4 and 5, we exposed subjects to stimulus differences that generally favored auditory loudness, the relevant dimension. In Experiment 6 , we asked subjects to perform unfilled and constant-fill tasks when stimulus differences were biased in favor of pitch. Because only two levels of stimulus quantity were tested, we expected the slopes of the Garner interference and linear congruity functions to be closer in magnitude to those observed in Experiment 4, which also tested two levels, than to those observed in Experiment 5, which tested three levels. Because the experimental design is more similar in stimulus-task uncertainty to those used in Experiments 4 and 5 than to those used in Experiments 2 and 3, we expected that linear congruity would be generally negative in sign, and would become more negative as the frequency difference increased.

\section{Method}

Subjects. Thirty-three Purdue undergraduates (17 men and 16 women) participated in partial fulfillment of a course requirement. None had participated in any other experiment in this study.

Stimuli, Apparatus, and Procedure. The stimulus set contained 20 sine-wave signals, 10 levels of frequency $(900-990 \mathrm{~Hz}$ in steps of $10 \mathrm{~Hz}$ ) at each of the two levels of intensity ( 60 and $70 \mathrm{~dB})$. From this set we created 11 loudness tasks: 1 baseline task (at $900 \mathrm{~Hz}$ ), 5 unfilled filtering tasks, and 5 constant-fill filtering tasks. The filtering tasks differed in frequency range and stimulus quantity. In each unfilled task, two levels of frequency were varied; one level was always $900 \mathrm{~Hz}$, and the other level was $950 \mathrm{~Hz}(50-\mathrm{Hz}$ unfilled task), $960 \mathrm{~Hz}(60-\mathrm{Hz}$ unfilled task $), 970 \mathrm{~Hz}(70-\mathrm{Hz}$ unfilled task), $980 \mathrm{~Hz}$ (80-Hz unfilled task), or $990 \mathrm{~Hz}(90-\mathrm{Hz}$ unfilled task). The baseline and each unfilled task contained 200 trials.

The constant-fill conditions were created in a manner slightly different from those in Experiment 5. We began with the $50-\mathrm{Hz}$ constant-fill task, in which each level of intensity appeared 16 times at each of 6 frequencies (192 total trials); the 6 frequency levels were $900-950 \mathrm{~Hz}$ in $10-\mathrm{Hz}$ steps. To create the other constant-fill tasks, one level of frequency was added for each $10-\mathrm{Hz}$ increment in range (i.e., $60,70,80$, and $90 \mathrm{~Hz}$ ). In the $90-\mathrm{Hz}$ constant-fill task, for example, each level of intensity appeared 10 times at each of 10 frequencies (200 total trials); the frequency levels were $900-990 \mathrm{~Hz}$ in $10-\mathrm{Hz}$ steps. Thus, the constant-fill conditions involved a slight increase in stimulus quantity as the frequency difference increased.

Order of presentation was determined by means of a Latin square. All other aspects duplicated those of the previous experiments.

\section{Results}

Overall performance. The subjects' overall inaccuracy was $4.06 \%$. The correlation between speed and accuracy was .92. An ANOVA of error percentages revealed a significant effect of task $\left[F(10,290)=15.67, M S_{\mathrm{e}}=2.98\right.$, $p<.001]$. Newman-Keuls analysis showed that the error rate in the $70-\mathrm{Hz}$ unfilled task $(6.48 \%)$, the $80-\mathrm{Hz}$ unfilled task $(6.32 \%)$, and the $90-\mathrm{Hz}$ unfilled task $(6.50 \%)$ was significantly larger than in other tasks, whereas the 


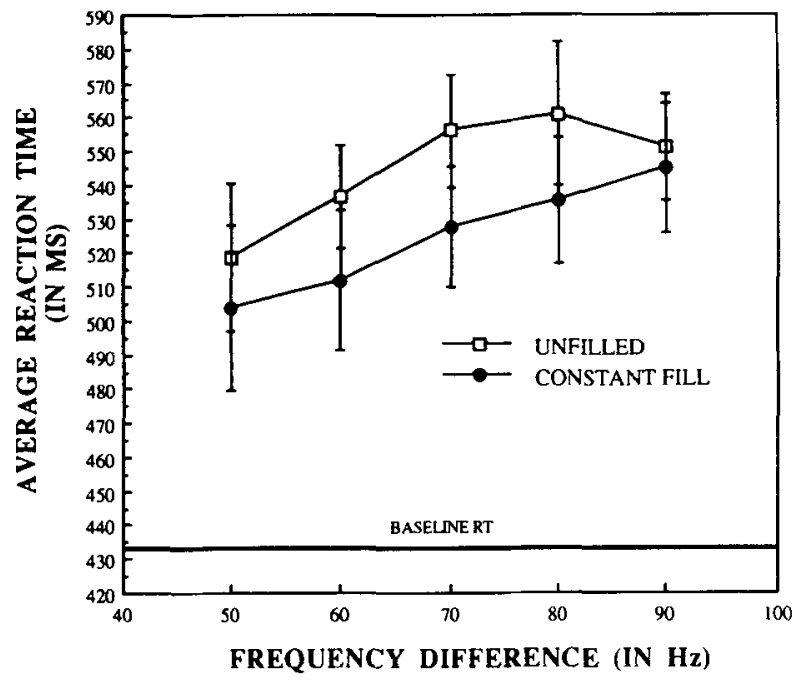

Figure 8. Average reaction time (RT, in milliseconds) in each of the 10 filtering tasks of Experiment 6. The parameter specifies stimulus quantity (unfilled, constant fill), and the abscissa specifies the difference in auditory frequency (in hertz) of values along the irrelevant dimension $(50,60,70,80$, and $90 \mathrm{~Hz})$. Note: Variability in each condition is shown by standard error bars. The bold horizontal line represents average RT performance at baseline.

error rate at baseline $(2.48 \%)$ and in the $50-\mathrm{Hz}$ constant task $(3.44 \%)$ was significantly smaller than in other tasks.

Garner interference. Average RTs at baseline and in each filtering task appear in Figure 8. An ANOVA of Garner interference was performed with pitch discriminability (five levels: $50,60,70,80$, and $90 \mathrm{~Hz}$ ) and stimulus quantity (two levels: filled and constant fill) as factors. There was a significant effect of discriminability $\left[F(4,128)=6.42, M S_{\mathrm{e}}=2,761.63, p<.001\right]$. Figure 8 shows that Garner interference increased steadily with increases in the frequency difference. There was also a significant effect of stimulus quantity $[F(1,32)=11.75$, $\left.M S_{\mathrm{e}}=2,809.53, p<.01\right]$, with Garner interference in the unfilled condition $(111 \mathrm{msec})$ exceeding that in the constant-fill condition $(91 \mathrm{msec})$. Finally, despite the noticeable decrease in the $90-\mathrm{Hz}$ unfilled task (see Figure 8), the interaction between frequency difference and stimulus quantity was not reliable $[F(4,128)=.48$, $M S_{\mathrm{e}}=2,839.76$, n.s.].

Linear congruity. The congruity effect in each filtering task, computed by subject and averaged, appears in Table 5. All congruity effects were significantly more negative than zero, as shown by $t$ test.

An ANOVA performed on congruity effects revealed a significant main effect of pitch discriminability $[F(4,128)$ $\left.=14.50, M S_{\mathrm{e}}=1,268.97, p<.001\right]$. Newman-Keuls post hoc analysis showed that congruity effects at each level of discriminability differed from one another, with the exception of $60 \mathrm{~Hz}$ versus $70 \mathrm{~Hz}$ and $80 \mathrm{~Hz}$ versus
$90 \mathrm{~Hz}$. There was also a main effect of stimulus quantity $\left[F(1,32)=8.13, M S_{\mathrm{e}}=1,712.91, p<.01\right]$, and a significant interaction between discriminability and quantity $\left[F(4,128)=2.78, M S_{\mathrm{e}}=1,011.816, p=.03\right]$. Interestingly, the average congruity effect in the constant-fill condition ( $-95 \mathrm{msec}$ ) was significantly greater than the average congruity effect in the unfilled condition $(-82 \mathrm{msec})$.

\section{Discussion}

The results of Experiment 6 were straightforward. Subjects suffered more Garner interference and displayed more negative linear congruity the larger the discriminability of the irrelevant dimension and the fewer the values within the range of the irrelevant dimension. These results conform well with our expectations, which were based on the other experiments in this study.

Experimental context played a significant role in Experiment 6 , as is evidenced by several findings. First, the slopes of the Garner interference functions (unfilled, .90 , $r^{2}=.68$; constant fill, 1.06, $r^{2}=.99$ ) were not as steep as those found in corresponding conditions of Experiment 5 (unfilled, 2.31, $r^{2}=1.00$; constant fill, 1.74, $r^{2}=.99$ ), where uncertainty was relatively high, but steeper than those obtained in Experiment 3 (unfilled, .68, $r^{2}=.87$ ), where uncertainty was relatively low. Also of interest was the sharp jump in the intercept: it was 48.57 in the unfilled condition, -13.77 in the same condition of Experiment 5, and 27.00 in Experiment 3. The change in intercept indicates that Garner interference depends, among other things, on the range of discriminabilities used in an experiment (cf. Gravetter \& Lockhead, 1973;

Table 5

Average Reaction Time (RT, in Milliseconds) to Congruent and Incongruent Stimuli and Linear Congruity Effects (LCEs) in Each of Six Filtering Tasks in Experiment 6

\begin{tabular}{|c|c|c|c|c|c|}
\hline \multirow[b]{3}{*}{ Condition } & \multicolumn{4}{|c|}{ Tone } & \multirow[b]{3}{*}{ LCE } \\
\hline & \multicolumn{2}{|c|}{ Soft } & \multicolumn{2}{|c|}{ Loud } & \\
\hline & Low (C) & High (I) & Low (I) & High (C) & \\
\hline $50-\mathrm{Hz}$ unfilled & 558 & 504 & 463 & 537 & -64 \\
\hline 50-Hz constant & 535 & 488 & 458 & 533 & -61 \\
\hline $60-\mathrm{Hz}$ unfilled & 575 & 513 & 477 & 582 & -84 \\
\hline $60-\mathrm{Hz}$ constant & 550 & 489 & 443 & 554 & -87 \\
\hline $70-\mathrm{Hz}$ unfilled & 589 & 537 & 498 & 600 & -77 \\
\hline $70-\mathrm{Hz}$ constant & 561 & 492 & 448 & 574 & -98 \\
\hline 80-Hz unfilled & 589 & 529 & 487 & 604 & -88 \\
\hline 80-Hz constant & 589 & 495 & 459 & 599 & -117 \\
\hline 90-Hz unfilled & 601 & 519 & 487 & 596 & -95 \\
\hline $90-\mathrm{Hz}$ constant & 602 & 506 & 479 & 604 & -111 \\
\hline
\end{tabular}

Note-Stimulus RTs were estimated from linear regression analyses of response classification functions (see text for details). The columns labeled Low represent stimuli with a frequency of $900 \mathrm{~Hz}$. The columns labeled High represent stimuli whose frequency is the sum of $900 \mathrm{~Hz}$ and the frequency of the particular condition (e.g., High in the $60-\mathrm{Hz}$ condition is $960 \mathrm{~Hz}$ ). LCE was computed by subtracting the average RT to congruent stimuli (low-pitched soft tones and high-pitched loud tones) from the average RT to incongruent stimuli (high-pitched soft and low-pitched loud tones). $C$ and I denote congruent and incongruent stimuli, respectively. 
Marks, 1988). In this study, the interference function shifted upward as the task milieu changed from discriminabilties consistently below the matching point (Experiments 4 and 5), to balanced discriminabilities (Experiment 3 ), to discriminabilities consistently above the matching point (Experiment 6). In this regard it is worth noting that, overall, Garner interference averaged $111 \mathrm{msec}$ in the unfilled tasks of Experiment 6; it was only $71 \mathrm{msec}$ in the corresponding tasks of Experiment 3. Obviously, the exact same tasks can lead to very different degrees of interactive processing, depending on the experimental context in which they are performed.

The power of uncertainty was also evident in the pattern of linear congruity. Congruity effects were strongly negative in this experiment, coinciding with the results of congruity analyses in Experiments 4 and 5, but contrasting with results from Experiments 2 and 3. Intercepts, especially in the unfilled condition $\left(-34.22, r^{2}=.80\right)$, were also strongly negative. Again, it is instructive to compare the results of Experiment 6 with the corresponding tasks of Experiment 3. The average congruity effect in the 50-, 60-, 70-, 80-, and 90-Hz (unfilled) tasks of Experiment 3 was $+38 \mathrm{msec}$. The average congruity effect in the identical tasks performed in Experiment 6 was $-82 \mathrm{msec}$, reflecting a change in sign and an increase in absolute magnitude. As with Garner interference, the exact same tasks led to very different patterns of linear congruity depending on the uncertainty level.

\section{GENERAL DISCUSSION}

Six experiments were conducted to illuminate multidimensional processing in auditory perception. Subjects classified sounds as loud or soft, and attempted to ignore orthogonal variation in auditory pitch. In Experiment 1 we found that, on the average, RT for discriminating sounds that differed by $10 \mathrm{~dB}$ at a constant frequency was approximately equal to $\mathrm{RT}$ for discriminating sounds that differed by $50 \mathrm{~Hz}$ at a constant intensity. In Experiment 2 we found that as the number of pitch values in a range increased, Garner interference decreased, but linear congruity remained relatively constant. In Experiment 3, we found that both Garner interference and linear congruity increased monotonically as the frequency range expanded from 10 to $100 \mathrm{~Hz}$.

Experiment 4 combined the two lower order contextual variables in a single session. This caused an increase in the slopes and a decrease in the intercepts relating discriminability to Garner interference. It also caused an astounding shift in linear congruity, from strongly positive in earlier experiments to strongly negative. This congruity shift was replicated in Experiment 5, in which a new level of stimulus quantity was added to the tasks tested in Experiment 4. Adding this condition caused further enhancement in the slopes and intercepts of Garner interference functions. Finally, in Experiment 6, we again manipulated the lower order contexts simultaneously, but at levels of pitch discriminability that were at or greater than the matching point of $50 \mathrm{~Hz}$. We found strong effects of
Garner interference and (negative) linear congruity which were affected linearly by increases in pitch discriminability; both performance functions were characterized by intercepts of large absolute value.

\section{A Summary Through Regression Analyses}

We have argued that interactive processing arises from the effects of context-setting variation along an irrelevant dimension on the classification of attributes from a relevant dimension. In the present study, we have examined the effects of three kinds of pitch contextual relations on loudness classification. One way to organize this set of findings is to identify the contribution of each contextual variable through regression models. With few exceptions, the changes in loudness classification wrought by pitch context, as measured in this study by Garner interference and linear congruity, were described well by linear functions. Of course, this linearity may be a partial consequence of the specific range of discriminabilities that we tested. Nonetheless, we sought to summarize the influences of context on interactive processing through a series of linear regressions.

We analyzed separately the average Garner interference and the average linear congruity across the various conditions of Experiments 2-6; there were 41 data points for each measure. In each model, the predictors were (1) relative discriminability, (2) stimulus quantity, and (3) stimulus-task uncertainty. The first two variables were defined continuously. The third variable was defined categorically in each of two ways: according to the experiment, with Experiments 2 and 3 together constituting one level and the remaining experiments each specifying its own level (i.e., four total levels); and dichotomously, with Experiments 2 and 3 together creating one level and Experiments 4,5 , and 6 together creating the other level. The categorical variable was allowed to interact with each of the continuous variables. We were most interested in determining whether a statistical interaction obtains between uncertainty and discriminability, as was hypothesized in the discussion of Experiment 4.

Garner interference was explained well by both of the regression models. When uncertainty was assigned four levels, the model acccounted for .96 of the total variance $\left(M S_{\mathrm{e}}=50.21\right)$. Statistically, there was a significant effect of discriminability $[F(1,26)=100.13, p<.001]$, a significant effect of stimulus quantity $[F(1,26)=8.46$, $p<.01]$, and a significant interaction between discriminability and uncertainty $[F(3,26)=5.03, p<.01]$. The smaller model, in which uncertainty was assigned only two levels, did almost as well, accounting for .92 of the total variance $\left(M S_{e}=136.63\right)$. Again, there was a significant effect of discriminability $[F(1,34)=160.49$, $p<.001]$ and a significant effect of stimulus quantity $[F(1,34)=7.63, p<.01]$. And, as hypothesized, a significant interaction was obtained between discriminability and uncertainty $[F(1,34)=15.41, p<.001]$. No other effects were reliable in either model.

The interaction effect from the smaller model is depicted in panel A of Figure 9. As one can see, the experimental 

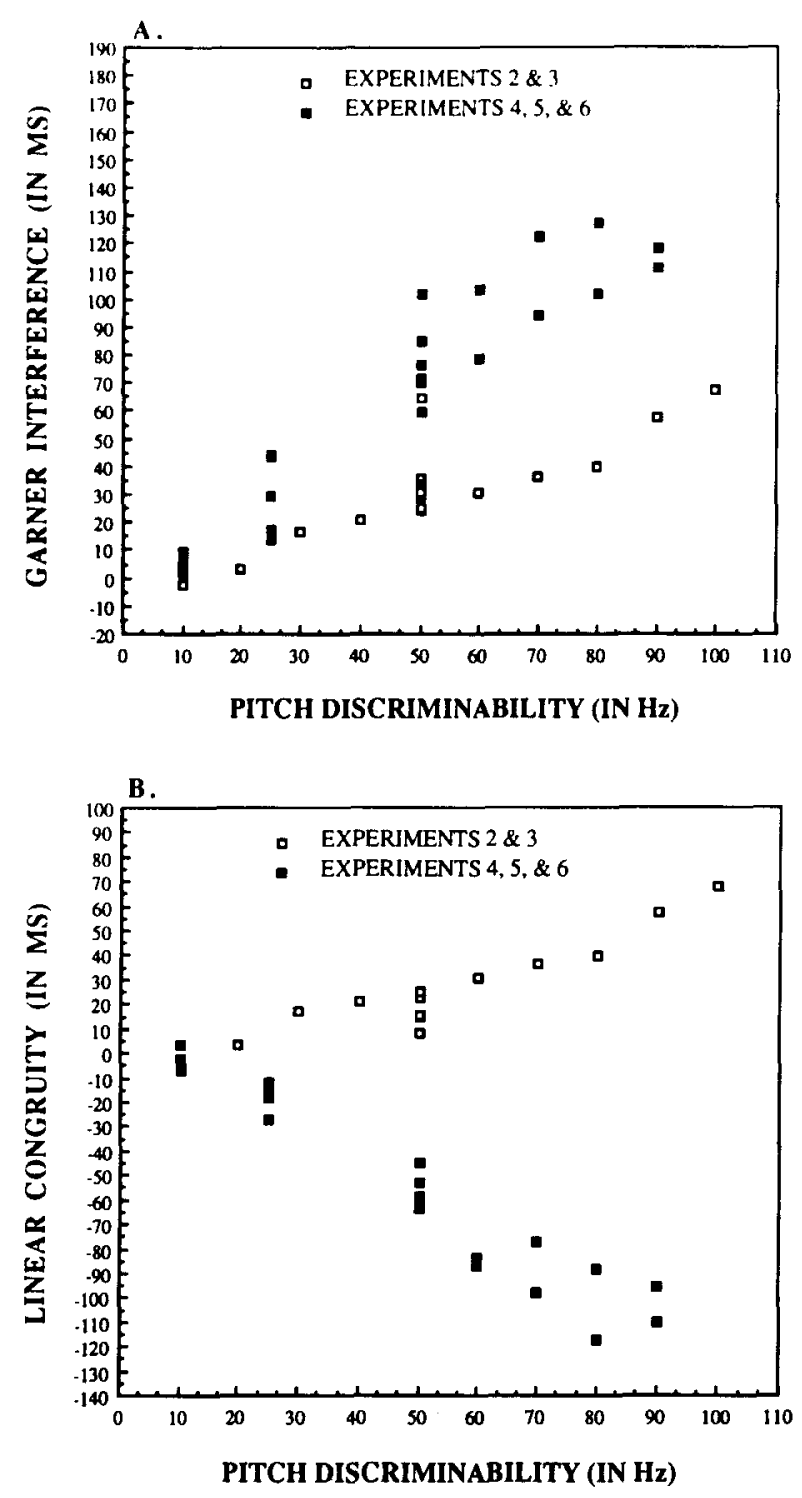

Figure 9. Interaction between stimulus-task uncertainty, specified by two levels (i.e., Experiments 2 and 3 vs. Experiments 4, 5 , and 6), and relative discriminability in pitch, specified by the frequency difference, in Garner interference (panel A) and linear congruity (panel B).

context created by Experiments 4, 5, and 6 led to a Garner interference function with a substantially steeper slope than did the experimental context created by Experiments 2 and 3 . The importance of the uncertainty variable can also be appreciated by comparing the fit of the two models tested here with that of a regression model containing only discriminability and quantity as predictors. The latter model, though significant statistically $[F(2,38)=24.70$, $p<.001]$, accounted for only .57 of the total variance. It is clear that all three contextual factors are needed to capture the present pattern of results.

Linear congruity was best described by a slightly different set of predictors. Both regression models were ex- cellent at accommodating our congruity data. The larger model, containing four levels of uncertainty, accounted for .99 of the total variance $\left(M S_{\mathrm{e}}=50.21\right)$. For this model, there was a marginal effect of uncertainty $[F(3,26)$ $=2.64, p=.07]$ and a significant effect of discriminability $[F(1,26)=48.04, p<.001]$. There was a significant interaction between uncertainty and discriminability $[F(3,26)=24.05, p<.001]$. Again, the smaller model did nearly as well, accounting for .97 of the total variance $\left(M S_{\mathrm{e}}=71.80\right)$. In the latter model, the effect of uncertainty was reliable $[F(1,34)=5.05, p=.03]$; there was also a significant effect of discriminability $[F(3,26)$ $=24.05, p<.001]$ and a significant interaction between uncertainty and discriminability. Quantity was not a reliable predictor in either model, a finding in keeping with our earlier discussions.

The interaction between uncertainty and discriminability in the smaller model is depicted in panel B of Figure 9. As one can see, the experimental context created by Experiments 2 and 3 had one effect on linear congruity, while the experimental context created by Experiments 4, 5, and 6 had the inverse effect. The importance of uncertainty to linear congruity is most strongly indicated by the poor fit of a regression model that removes the uncertainty variable. This model was not significant statistically $[F(2,38)$ $=1.13$, n.s.], and accounted for only .06 of the total variance.

\section{The Contextual Control of Dimensional Interaction}

From these summary models, we can begin to piece together the role of contextual relations in multidimensional processing. The results indicate clearly that dimensional interaction is not all or none. Interactive processing can be slight or great, depending on both higher order and lower order contextual factors. The results suggest that these factors might even completely control selective attention: context can alter the magnitude, the rate, and even the direction of either stimulus effects (linear congruity) or task effects (Garner interference). As the regression models confirm, these contextual factors operate in a graded and lawful manner.

The regression analyses indicate that the effects of uncertainty on classification are largely dichotomous; our data were explained well by just two levels of uncertainty: (1) situations in which quantity and discriminability were manipulated separately (Experiments 2 and 3 ) versus (2) situations in which these stimulus factors were manipulated jointly (Experiments 4, 5, and 6). But although these two levels captured the changes in slope seen in individual performance functions, the models may have obscured concomitant changes that we observed in the intercepts of these functions (e.g., by averaging the negative intercepts of Experiments 4 and 5 with the positive intercepts of Experiment 6).

With respect to the two lower order contexts, we found that discriminability brings the strongest influences to bear on interactive processing, both alone and as mediated by uncertainty. The latter finding hints that humans process 
very complex relations among the different sources of contextual information (i.e., the different types of dimensional variation). The effects of stimulus quantity were largely restricted to Garner interference. Hence, stimulus quantity serves to dissociate Garner interference from linear congruity; apparently, these two measures are not interchangeable.

Each of the three types of context studied here is defined by the manner in which values from the irrelevant dimension (pitch) changed from trial to trial. Although our results are consistent with many different theoretical viewpoints, one way to conceptualize the role of these trial-to-trial changes is as undermining the perceptual resolution of loudness. If one imagines quadrilaterals represented in psychological space, the spatial metaphor employed throughout this article (see Figure 2), then one might consider that increasing rectangle height (i.e., pitch discriminability) or decreasing its vertical density (i.e., pitch quantity) causes a shrinkage in rectangle length (i.e., loudness resolution). The strong influences of uncertainty observed throughout this study indicate that loudness resolution is also greatly affected by task milieu. Given our data, loudness differences would seem to shrink continuously as each contextual factor is manipulated.

We doubt, though, that perceived differences can be made infinitely small. We would expect, for example, that a Garner interference function would flatten as pitch discriminability became extreme; otherwise, one would be forced to predict that, under certain pitch contexts, loudness discrimination was impossible. Indeed, there was some suggestion in Experiment 6 that Garner interference in the unfilled condition and linear congruity in the constant-fill condition approached asymptote at higher pitch discriminabilities (see Figure 8 and Table 5). We must await further research, however, before identifying whether and what boundaries limit the effects of context on multidimensional classification.

But even if we assume that strict limits on context exist, the present study illustrates that multidimensional processing is powerfully, perhaps even fully, controlled by the contexts of dimensional variation. The clear indication is that interactive effects are mainly a characteristic of stimulus relations and stimulus changes, rather than a quality intrinsic to a pair of dimensions. This is not to deny the traditional distinction between interacting and separable dimensions. We suggest, though, that only by understanding the different types and effects of contextual relations can one begin to characterize the nature of interaction or separability between dimensions.

\section{REFERENCES}

Algom, D., \& MarKs, L. E. (1990). Range and regression, loudness scales and loudness processing: Toward a context-bound psychophysics. Journal of Experimental Psychology: Human Perception \& Performance, 16, 706-721.

ASHay, F. G., \& TowNSEND, J. T. (1986). Varieties of perceptual independence. Psychological Review, 93, 154-179.

Carvellas, T., \& Schneider, B. (1972). Direct estimation of multi- dimensional tonal dissimilarity. Journal of the Acoustical Society of America, 51, 1839-1848.

Chipman, S. F., \& CAREY, S. (1975), Anatomy of a stimulus domain: The relation between multidimensional and unidimensional scaling of noise bands. Perception \& Psychophysics, 17, 417-424.

Demany, L. (1983). Speeded discrimination of frequency and intensity differences. Proceedings of the 11th International Congress on Acoustics, 3, 107-110.

Durlach, N. I., \& Braida, L. D. (1969). Intensity perception: I. Preliminary theory of intensity resolution. Journal of the Acoustical Society of America, 46, 372-383.

Eriksen, C. W., O'Hara, W. P., \& Eriksen, B. (1982). Response competition in same-different judgments. Perception \& Psychophysics, 32, 261-270.

FELFOLDY, G. L. (1974). Repetition effects in choice reaction time to multidimensional stimuli. Perception \& Psychophysics, 15, 453-459.

Foard, C. F., \& Kemler Nelson, D. G. (1984). Holistic and analytic modes of processing: The multiple determinants of perceptual analysis. Journal of Experimental Psychology: General, 113, 94-111.

GARNER, W. R. (1974). The processing of information and structure. Potomac, MD: Erlbaum.

Gravetter, F., Lockhead, G. R. (1973). Criterial range as a frame of reference for stimulus judgment. Psychological Review, 80, 203-216.

GREEN, D. M., SWETS, J. A. (1966). Signal detection theory and psychophysics. New York: Wiley.

Helson, H. (1964). Adaptation-level theory: An experimental and systematic approach to behavior. New York: Harper \& Row.

Holland, M. K. LocKHEAd, G. R. (1968). Sequential effects in absolute judgments of loudness. Perception \& Psychophysics, 3, 409-414.

LiNK, S. W. (1992). The wave theory of difference and similarity. Hillsdale, NJ: Erlbaum.

LOCKHEAD, G. R. (1992). Psychophysical scaling: Judgments of attributes or objects? Behavionial \& Brain Sciences, 15, 543-601.

MACLeOd, C. M. (1991). Half a century of research on the Stroop effect: An integrative review. Psychological Bulletin, 109, 163-203.

MADDOX, W. T. (1992). Perceptual and decisional separability. In F. G. Ashby (Ed.), Multidimensional models of perception and cognition (pp. 147-180). Hillsdale, NJ: Erlbaum.

MARKS, L. E. (1988). Magnitude estimation and sensory matching. Perception \& Psychophysics, 43, 511-525.

MARKS, L. E. (1992). The slippery context effect in psychophysics: Intensive, extensive, and qualitative continua. Perception \& Psychophysics, 51, 187-198.

MARKS, L. E., \& WARNER, E. (1991). Slippery context effects and critical bands. Journal of Experimental Psychology: Human Perception \& Performance, 17, 986-996.

Melara, R. D., Marks, L. E. (1990a). HARD and sofT interacting dimensions: Differential effects of dual context on classification. Perception \& Psychophysics, 47, 307-325.

Melara, R. D., \& Marks, L. E. (1990b). Perceptual primacy of dimensions: Support for a model of dimensional interaction. Journal of Experimental Psychology: Human Perception \& Performance, 16, $398-414$.

Melara, R. D., Marks, L. E., \& Lesko, K. E. (1992). Optional processes in similarity judgments. Perception \& Psychophysics, 51, 123-133.

Pomerantz, J. R. (1986). Visual form perception: An overview. In E. C. Schwab \& H. C. Nusbaum (Eds.), Pattern recognition by humans and machines: Visual perception (pp. 1-30). New York: Academic Press.

Redding, G. M., \& Tharp, D. A. (1981). Processing line location and orientation. Joumal of Experimental Psychology: Human Perception \& Performance, 7, 115-129.

SCHNEIDER, B., \& PARKER, S. (1990). Does stimulus context affect loudness or only loudness judgments? Perception \& Psychophysics, 48, 409-418.

Smith, J. D., \& Kemler Nelson, D. G. (1984). Overall similarity in adults' classification: The child in all of us. Journal of Experimental Psychology: General, 113, 137-159. 
WARD, L. M., \& LoCkHEAD, G. R. (1971). Response system processes in absolute judgment. Perception \& Psychophysics, 9, 73-78.

Wright, R. D. (1986). Amiga 1000 hardware timing and reaction-time key interfacing. Behavior Research Methods, Instruments, \& Computers, 18, 463-465.

ZAGORSKI, M. (1975). Perceptual independence of pitch and loudness in a signal detection experiment: A processing model for 2ATFC (21FC) experiments. Perception \& Psychophysics, 17, 525-531.

\section{NOTES}

1. We do not assume that the perceptual or response effects of a particular stimulus are invariant either across repeated presentations (Green \& Swets, 1966) or for the same presentation at different temporal points during a trial (Link, 1992). The stimuli are represented as points in multidimensional space solely for purposes of description. It is conceivable that the perceptual effects for a given level of loudness will change with the level of pitch, either in mean or in variance (e.g., Ashby \& Townsend, 1986). We do not distinguish these effects in the present paper, but use the spatial metaphor as a way of summarizing our manipulations and their consequences.

2. In this experiment and in succeeding experiments, we designed most of our filtering tasks around signals of $900 \mathrm{~Hz}$. It was appropriate, there- fore, that baseline discrimination also be performed at $900 \mathrm{~Hz}$. For ease of administration, and to facilitate comparisons across experiments, we used only the $900-\mathrm{Hz}$ task as baseline in each experiment. Results from Experiment 1, however, indicate a possible problem with this decision: loudness discrimination was fastest at this frequency, suggesting that a $900-\mathrm{Hz}$ baseline task might lead to slight overestimations of Garner interference. Fortunately, because our main interest is in the relative ordering of performance in filtering tasks, minor biases in estimations of absolute magnitude are inconsequential. Moreover, as we shall see, in each experiment subjects' speed at classifying low frequency stimuli in filtering tasks generally matched their speed at classifying highfrequency stimuli (i.e., never differing by more than approximately $20 \mathrm{msec}$ on the average). Furthermore, the average speed in classifying baseline stimuli varied little across experiments, despite the large shifts in stimulus-task uncertainty and the use of different subjects. All of these considerations imply that the benefits of maintaining the 900 $\mathrm{Hz}$ baseline across experiments greatly outweighed any slight errors of estimation.

(Manuscript received April 13, 1993; revision accepted for publication November 30, 1993.) 